\title{
International Energy Indicators
}

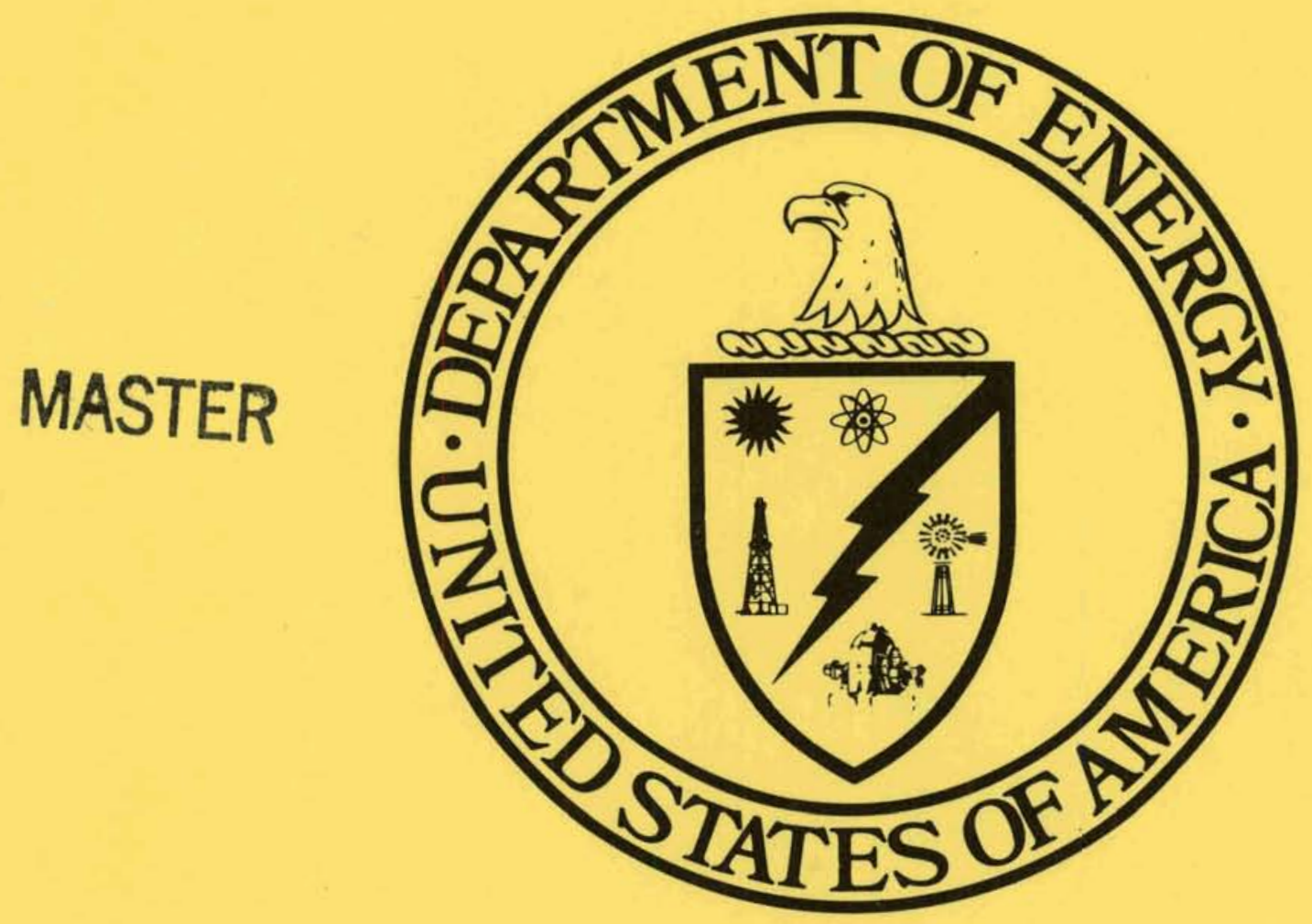

International Affairs

Office of Current Reporting

March 1980 


\section{DISCLAIMER}

This report was prepared as an account of work sponsored by an agency of the United States Government. Neither the United States Government nor any agency Thereof, nor any of their employees, makes any warranty, express or implied, or assumes any legal liability or responsibility for the accuracy, completeness, or usefulness of any information, apparatus, product, or process disclosed, or represents that its use would not infringe privately owned rights. Reference herein to any specific commercial product, process, or service by trade name, trademark, manufacturer, or otherwise does not necessarily constitute or imply its endorsement, recommendation, or favoring by the United States Government or any agency thereof. The views and opinions of authors expressed herein do not necessarily state or reflect those of the United States Government or any agency thereof. 


\section{DISCLAIMER}

Portions of this document may be illegible in electronic image products. Images are produced from the best available original document. 


\section{International Energy Indicators}

\section{DEPARTMENT OF ENERGY \\ International Affairs \\ Office of Current Reporting}

\section{March 1980}

Published monthly in the Office of Current Reporting, International Affairs.

Editor: Dr. Elizabeth K. Bauer, 252-5162, Forrestal Building

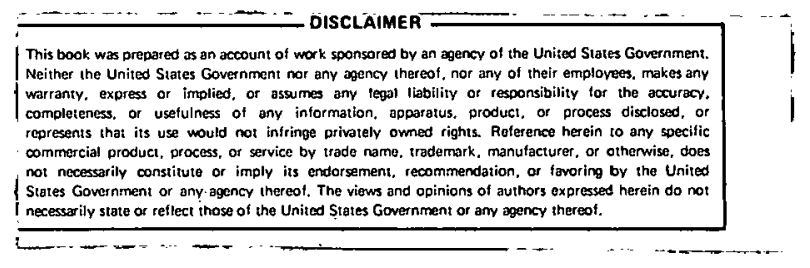


Estimated production rose slightly to $2.5 \mathrm{MMBD}$ in February, the averge concealing wide swings in output. Assuming that domestic consumption was about 700,000 BD, exports averaged around 1.8 MMBD.

Saudi Arabia: Crude Oil Capacity, Production and Shut-in, 1974 -

Saudi Arabia held its production at an estimated 9.8 MMBD in February. ARAMCO's temporary ceiling remeined at $9.5 \mathrm{MMBD}$, and .3 came from the Neutral Zone. The shut-in remained at zero.

OPEC (Ex-Iran and Saudi Arabia): Capacity, Production and Shut-in, 1974 The rest of OPEC was producing 17.28 MMBD in January, with about 1,975 MBD shut in.

Non-OPEC Free World and U.S. Production of Crude Oil, $1973-$

Non-OPEC crude oil production rose to $18.26 \mathrm{MMBD}$ in January. The bulk of the increase over the past 12 months (December 1978-December 1979) was in the North Sea and Mexico. U.S. production dropped by 182 MBD. Other Non-OPEC nations registered an increase of 202 MBD.

Oil Stocks: Freo World, U.S., Japan and Europe (Landed), 1973 -

Revised third quarter figures for the U.S., Japan and Europe (Big Five) show seasonal increases in preparation for winter. Other data not yet available.

Petroleum Consumption by Industrial Countries, 1973 -

The September-October figures show the normal seasonal pattern in consumption. The adjusted 12-month moving average for the 20 IEA countries plus France continued its upward trend. As indicated in the lower part of the graph, the moving average for the total ex-U.S. figures remains above the 1973 level. All 1979 data revised and updated.

USSR Crude Oil Production, 1974 -

The graphic display shows the rate of change of USSR oil production. The January and February figures, based on Soviet sources indicate a return to the downward trend. Annual export figures have been revised to show CIA estimates of total exports to both Bloc and Western countries.

Free World and U.S. Nuclear Generation Capacity, 1973 -

U.S. capacity was unchanged since November. Free World capacity dropped by $251 \mathrm{MW}$ in January, mainly because of the decommissioning of a 250-MW generator in West Germany. 
In January, total U.S. imports dropped slightly below 8.5 MMBD. The 12-month moving average, based on adjusted figures, was $8.0 \mathrm{MMBD}$.

\section{Landed Cost of Saudi Crude Oil in Current and 1974 Dollars -}

According to data derived from industry information provided to DOE and recently revised and updated, the landed cost of Saudi Light crude rose sharply to another new high of $\$ 22.99$ in October. In 1974 dollars, the real difference between April 1974 and October 1979 showed an increase of \$3.47, reflecting recent OPEC price increases and the beginnings of the "leap-frogging" operation that characterized the market at the end of 1979. More recent data are in preparation.

\section{U.S. Trade in Coal, $1973-$}

In December, exports were 6.7 million short tons of bituminous coal. Imports were 146,000 short tons of steam coal. The 12-month moving average for exports rose to 5.4 million short tons.

\section{Summary of U.S. Merchandise Trade, 1976 -}

The deficit rose to nearly $\$ 4.8$ billion in January from $\$ 3.1$ billion in December. Manufactured goods slipped into a slight deficit with only agriculture showing a surplus (\$1.6 billion). The deficit in energy was almost $\$ 6.3$ billion, close to the December figure.

\section{Energy/GNP Ratio -}

Fourth quarter 1979 preliminary figures follow the normal seasonal pattern. The moving average also dropped at a more or less constant rate.

NOTE: Annual figures from 1973 on and quarterly figures for 1978 and 1979 revised to conform to those shown in Monthly Energy Review for March. 
Iran: Crude Oil Capacity, Production and Shut-In, 1974 -

(In Thousands of Barrels per Day)

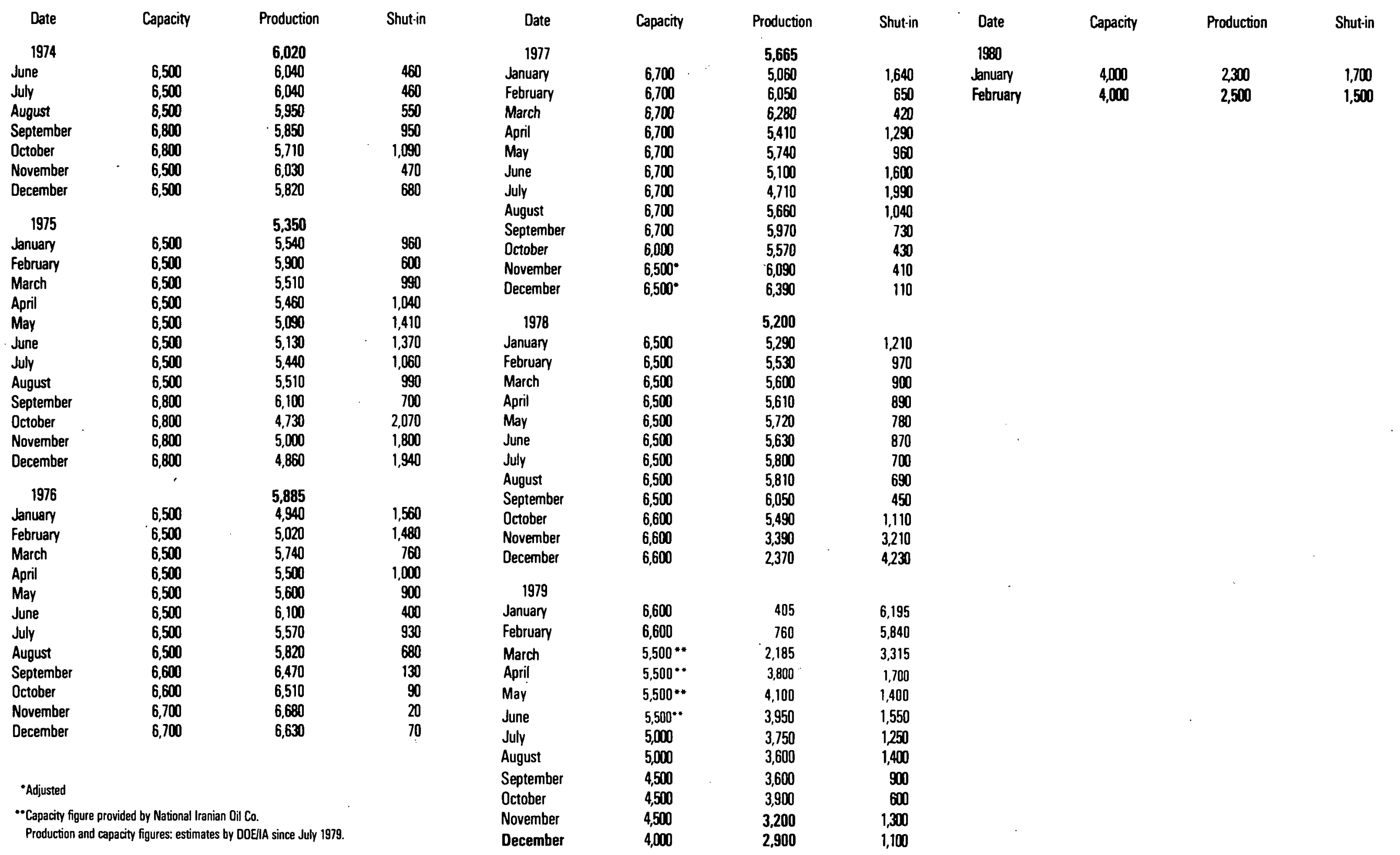


Iran-Capacity, Production and Shut-In, 1974 -

(In Millions of Barrels a Day)

MMBD

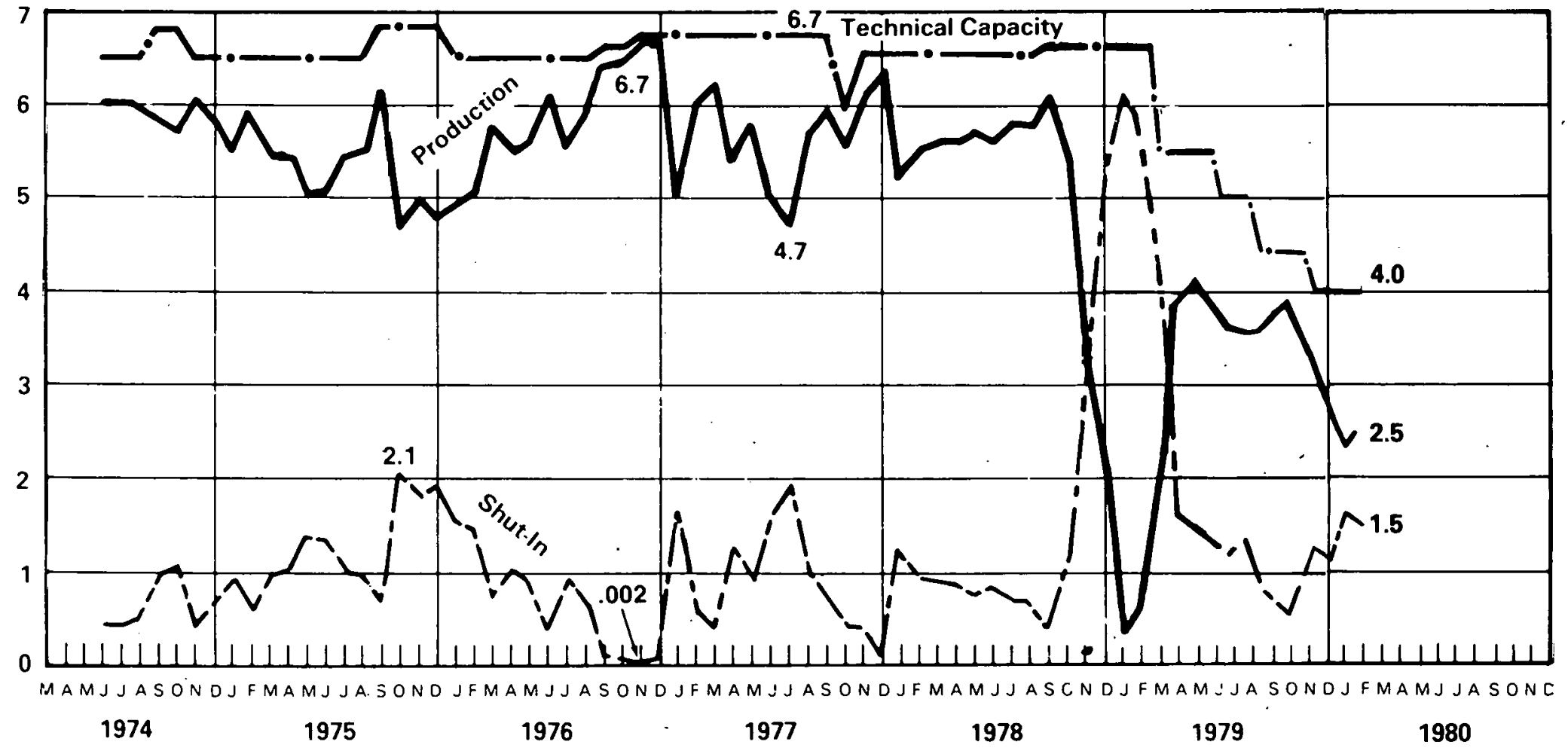

Source: CIA, Energy Branch 
Saudi Arabia: Crude Oil Capacity, Production and Shut-In, 1974 -

(In Thousands of Barrels per Day)

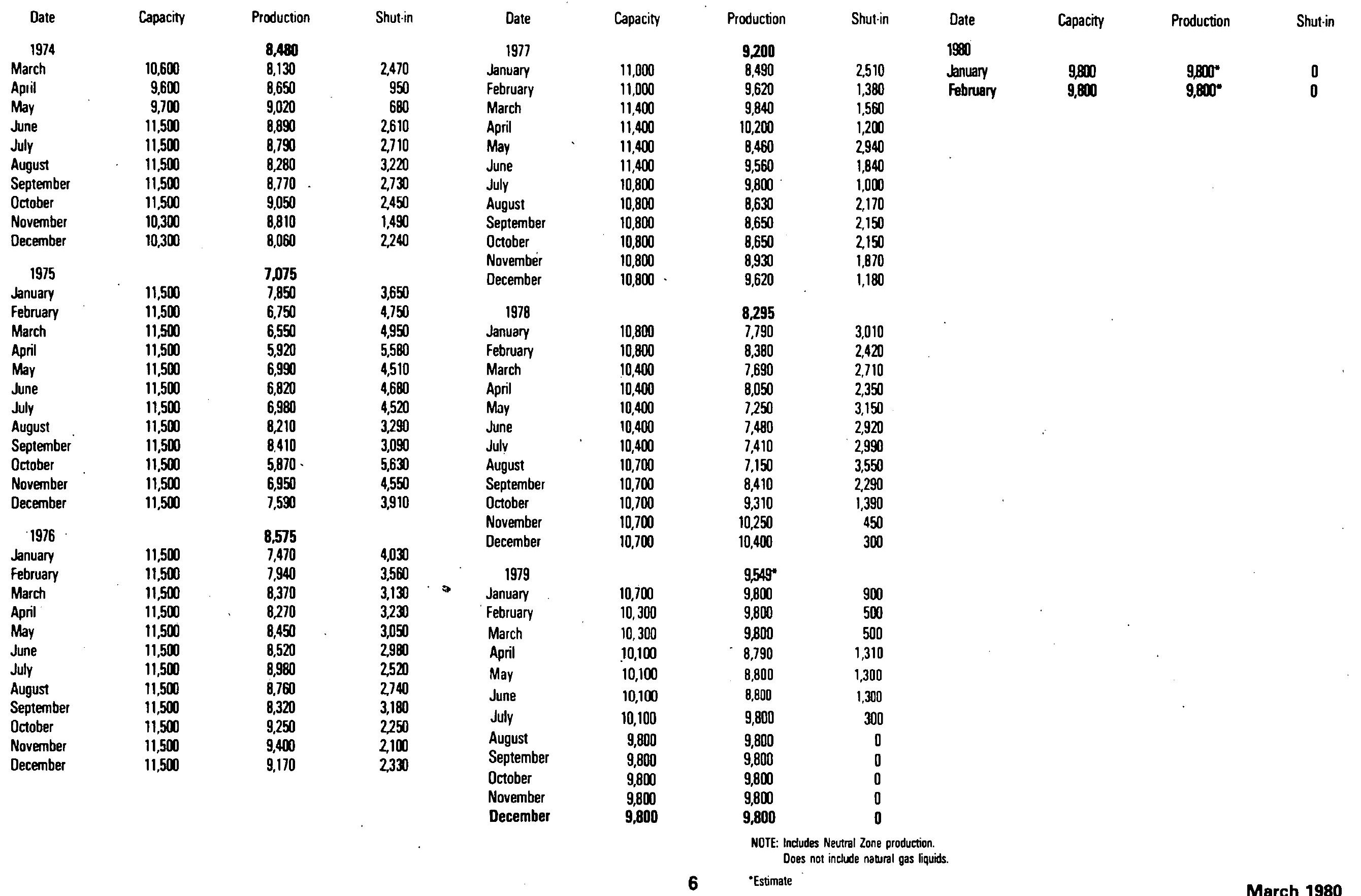


Saudi Arabia-Capacity, Production and Shut-In, 1974 -

(In Millions of Barrels a Day)

MMBD

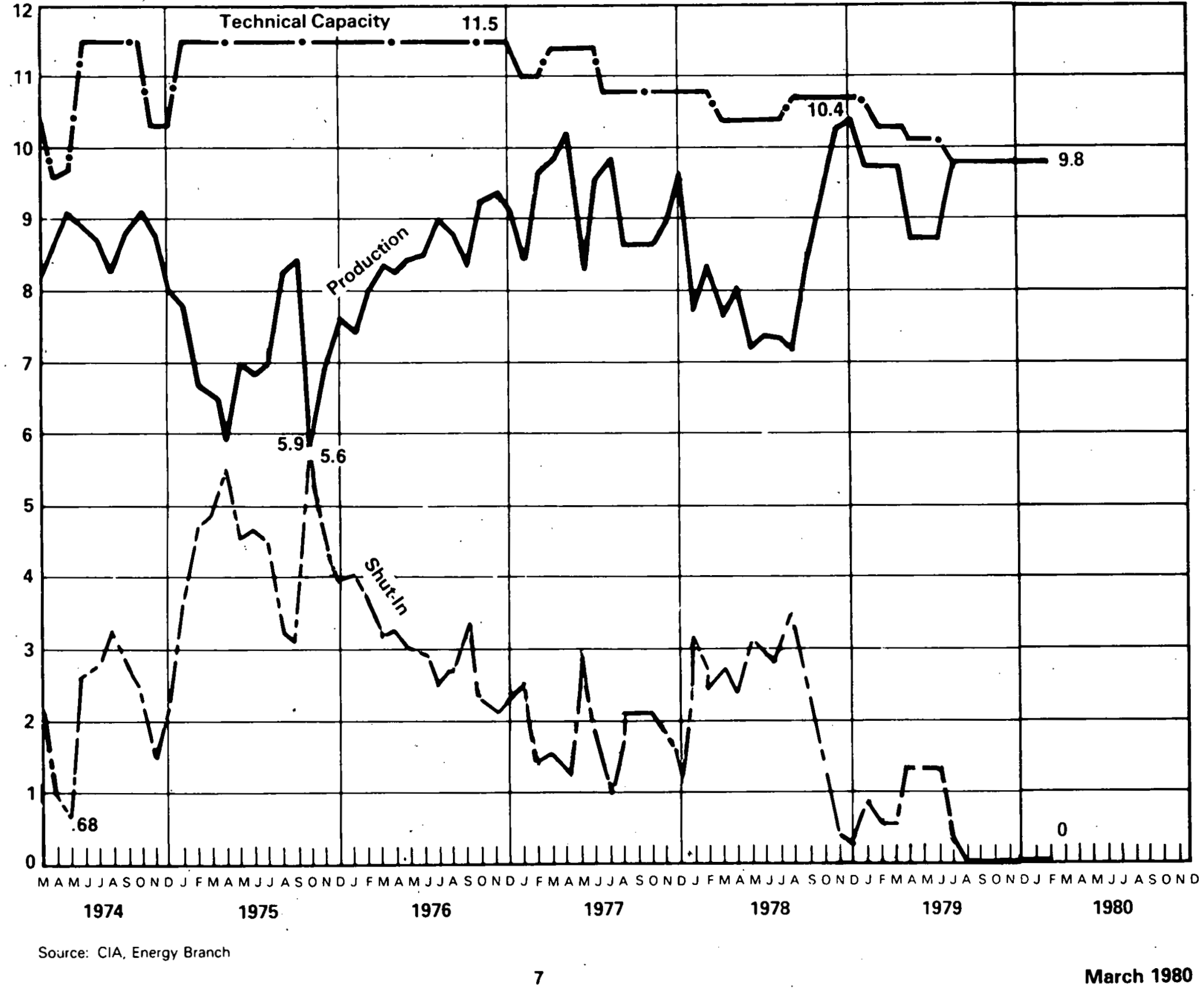


OPEC (Ex-Iran and Saudi Arabia): Capacity, Production and Shut-In, 1974 (In Thousands of Barrels a Day)

\begin{tabular}{|c|c|c|c|c|c|c|c|c|c|c|c|}
\hline Date & Capacity & Production & Shut-in & Date & Capacity & Production & Shut-in & Date & Capacity & Production & Shutin \\
\hline 1974 & & 16,175 & & $19 \pi$ & & 16,295 & & 1980 & & & \\
\hline June & 21,090 & 16,980 & 4,110 & January & 20.335 & 15,530 & 4,805 & January & 19,260 & 17,285 & 1,975 \\
\hline July & 20,740 & $15,67 n$ & 5.070 & February & 20,335 & 16,630 & 3,706 & & & & \\
\hline August & 20,740 & 15210 & 5.530 & March & 20,345 & 17,060 & 3285 & & & & \\
\hline September & 20,490 & 15,150 & 5,340 & April & 20,345 & 16,650 & 3,695 & & & & \\
\hline October & 20,490 & 14.720 & 5,770 & May & 20,345 & 16230 & 4.115 & & & & \\
\hline Hovember & 21,040 & 15,170 & 5,870 & June & 20.345 & 16290 & 4,055 & & & & \\
\hline December & 21,040 & 14,507 & 6.533 & July & 20,345 & 15,320 & 5,025 & & & & \\
\hline 1975 & & 14,710 & & September & 19,745 & 16,340 & 3,406 & & & & \\
\hline January & 20,840 & 13,780 & 7,060 & October & 19,645 & 15,980 & 3,665 & & & & \\
\hline February & 20,840 & 13280 & 7.560 & November & 19,695 & 16,740 & 2,955 & & & & \\
\hline March & 20,840 & 13.630 & 7210 & December & 19.685 & 16,910 & 2,785 & & & & \\
\hline April & 20,840 & 14,340 & 6.500 & & & & & & & & \\
\hline May & 20,840 & 13,990 & 6,850 & 1978 & & 16.120 & & & & & \\
\hline June & 20,840 & 14.720 & 6.120 & January & 19.645 & 14,320 & 5,325 & & & & \\
\hline July & 20,990 & 15.680 & 5,310 & February & 19,645 & 14,590 & 5,055 & & & & \\
\hline August & 20,990 & 15,450 & 5,540 & March & 19.645 & 15,080 & 4,565 & & & & \\
\hline October & 20,740 & 14.570 & 6.170 & May & 19,705 & 14,930 & 4,775 & & & & \\
\hline November & 20,740 & 15,110 & 5.630 & June & 19,705 & 15,570 & 4,135 & & & & \\
\hline \multirow[t]{2}{*}{ December } & 20,740 & 15,100 & 5,640 & July & 19,705 & 15,750 & 3,955 & & & & \\
\hline & & & & August & 19,705 & 16,325 & 3,380 & & & & \\
\hline 1976 & & 16,195 & & September & 19280 & 17,350 & 1,930 & & & & \\
\hline January & 20,590 & 14200 & 6,390 & October & 19200 & 16,700 & 2.500 & & & & \\
\hline February & 20,590 & 15,330 & 5260 & Hovember & 19200 & 17,870 & 1,330 & & & & \\
\hline March & 20,610 & 15,270 & 5,340 & December & 19.360 & 17,540 & 1.820 & & & & \\
\hline April & 20.610 & 15,310 & 5300 & & & & & & & & \\
\hline May & 20,610 & 15,360 & 5250 & 1979 & & $17,680^{*}$ & & & & & \\
\hline June & 20,430 & 15,960 & 4,470 & January & 19,360 & 18.115 & 1245 & & & & \\
\hline July & 20,430 & 15,800 & 4,630 & February & 18.960 & 18.270 & 690 & & & & \\
\hline August & 20,430 & 16,060 & 4,370 & March & 18,960 & 18,000 & 960 & & $\cdot$ & & \\
\hline September & 20,535 & 17,040 & 3.496 & April & 18,960 & 17,550 & 1,430 & & & & \\
\hline October & 20,155 & 17,360 & 2,796 & May & 18,960 & 17,760 & 1,200 & . & & & \\
\hline November & 20,155 & 17,060 & 3,095 & June & 18,960 & 17,740 & 1,220 & & & & \\
\hline December & 20,180 & 18260 & 1,920 & July & 19,260 & 17,595 & 1,665 & & & : & \\
\hline \multirow{2}{*}{\multicolumn{4}{|c|}{$\begin{array}{l}\text {-Petroleum Inteligence Weekly Estimate } \\
\text { Estimates: Petroleum Intelligence Weekly }\end{array}$}} & August & 19,260 & 17,150 & 2,110 & & & & \\
\hline & & & & December & 19,260 & 17,660 & 1,600 & & & & \\
\hline
\end{tabular}


OPEC (Ex-Iran and Saudi Arabia) Capacity, Production and Shut-In, 1974-

(In Millions of Barrels per Day)

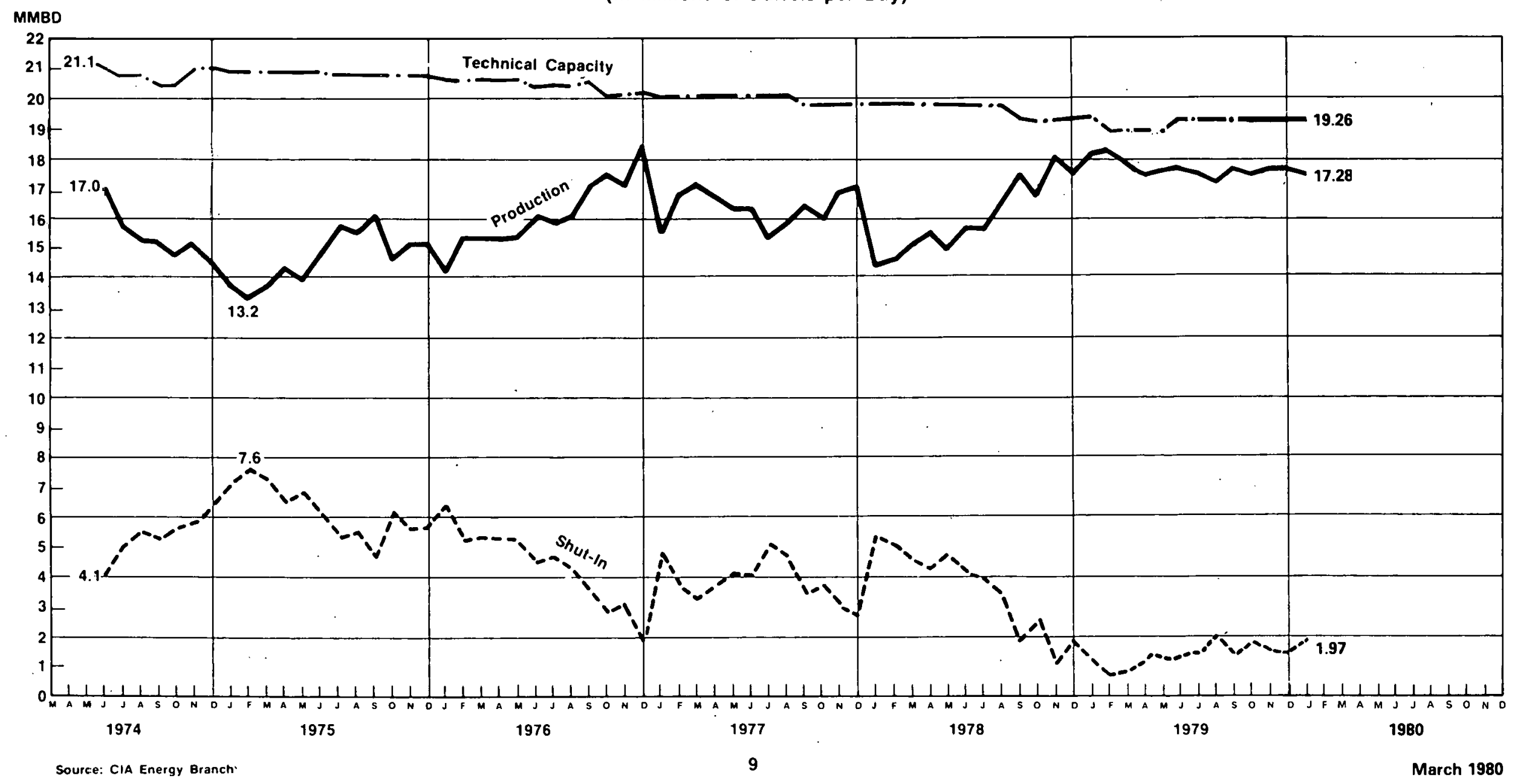


Non-OPEC Free World and U.S: Production of Crude Oil, 1973 (In Thousands of Barrels a Day)

\begin{tabular}{lcc} 
Month & Non-OPEC & US \\
Production & Production \\
January & & \\
February & 14,930 & 9,180 \\
March & 15,160 & 9,400 \\
April & 15,080 & 9,270 \\
May & 15,160 & 9,290 \\
June & 15,070 & 9,260 \\
July & 14,960 & 9,210 \\
August & 15,120 & 9,220 \\
September & 15,080 & 9,170 \\
October & 14,860 & 9,070 \\
November & 14,750 & 9,220 \\
December & 14,660 & 9,160 \\
AVERAGE & 14,660 & 9,060 \\
\multicolumn{1}{c}{1974} & 14,957 & 9,210 \\
January & & \\
February & 14,700 & 8,930 \\
March & 15,040 & 9,140 \\
April & 14,860 & 8,970 \\
May & 14,730 & 8,950 \\
June & 14,690 & 8,910 \\
July & 14,540 & 8,780 \\
August & 14,520 & 8,780 \\
September & 14,410 & 8,700 \\
October & 14,330 & 8,440 \\
November & 14,490 & 8,610 \\
December & 14,270 & 8,570 \\
AVERAGE & 14,480 & 8,530 \\
\multicolumn{1}{|c|}{1975} & 14,588 & 8,770 \\
January & & \\
February & 14,350 & 8,440 \\
March & 14,500 & 8,580 \\
April & 14,300 & 8,480 \\
May & 14,100 & 8,440 \\
June & $14,, 170$ & 8,370 \\
& 14,550 & 8,410 \\
& &
\end{tabular}

\begin{tabular}{|c|c|c|}
\hline Month & $\begin{array}{l}\text { Non-OPEC } \\
\text { Production }\end{array}$ & $\begin{array}{c}\text { US } \\
\text { Production }\end{array}$ \\
\hline \multicolumn{3}{|l|}{1975} \\
\hline July & 14,590 & 8,330 \\
\hline August & 14,350 & 8,240 \\
\hline September & 14,350 & 8,270 \\
\hline October & 14,420 & 8,310 \\
\hline November & 14,270 & 8,270 \\
\hline December & 14,550 & 8,240 \\
\hline AVERAGE & 14,367 & 8,375 \\
\hline \multicolumn{3}{|l|}{1976} \\
\hline January & 14,230 & 8,210 \\
\hline February & 14,250 & 8,200 \\
\hline March & 14,050 & 8,180 \\
\hline April & 13,900 & 8,080 \\
\hline May & 14,250 & 8,170 \\
\hline June & 14,520 & 8,140 \\
\hline July & 14,530 & 8,100 \\
\hline August & 14,500 & 8,070 \\
\hline September & 14,570 & 8,190 \\
\hline October & 14,580 & 8,050 \\
\hline November & 14,660 & 8,040 \\
\hline December & 14,730 & 8,010 \\
\hline AVERAGE & 14,397 & 8,132 \\
\hline \multicolumn{3}{|l|}{1977} \\
\hline January & 14,620 & 7,790 \\
\hline February & 15,100 & 8,070 \\
\hline March & 15,200 & 8,020 \\
\hline April & 14,950 & 8,080 \\
\hline May & 15,150 & 8,010 \\
\hline June & 15,410 & 8,040 \\
\hline July & 15,080 & 8,040 \\
\hline August & 15,560 & 8,240 \\
\hline September & 15,610 & 8,420 \\
\hline October & 15,860 & 8,520 \\
\hline November & 16,810 & 8,460 \\
\hline $\begin{array}{l}\text { December } \\
\text { Decmber }\end{array}$ & 16,350 & 8,540 \\
\hline $\bar{A} \bar{A} \bar{E} \bar{R} A G \bar{E}$ & 15,475 & 8,245 \\
\hline
\end{tabular}

Non-OPEC

Production

US

Production

1978

15,830

8,360

February

16,060

16,470

16,740

16,770

17,120

16,890

16,962

16,716

16,910

17,180

17,460

16,759

8,377

8,720

8,818

8,825

8,832

8,756

8,758

8,800

8,820

8,741

8,662

8,707

AVERAGE

17,444

17,515

8,457

January

February

17,440

8,498

April

May

June

July

August

September

October

November

December

17,555

17,520

17,650

17,820

17,735

17,605

17,780

17,965

17,965
18,110

17,678

1980

January*

18,262

8,585

8,533
8,585

8,409

8,355

8,699

8,510

8,460

8,530

8,480

8,505

8,459

Sources: CIA Energy Brench

Monthy Energy Review

"Preliminary estimates, Petroleum Intelligence Weekly 
Non-OPEC Free World and U.S. Production of Crude Oil ,1973 -

MMBD

(In Millions of Barrels a Day)

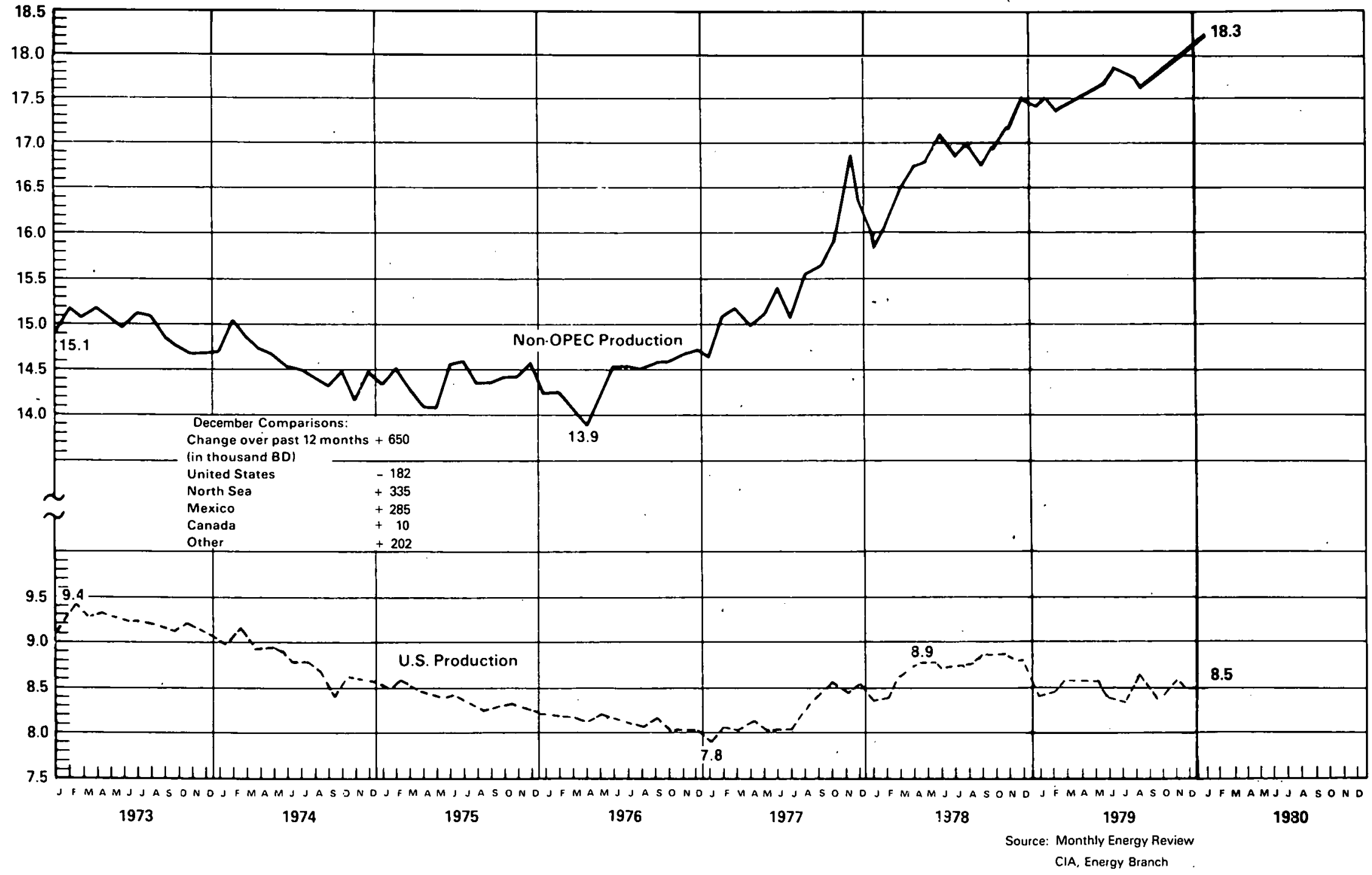

March 1980 
Oil Stocks: Free World, U.S., Japan and Europe (Landed, 1973 (In Million Barrels, End of Quarter)

\begin{tabular}{|c|c|c|c|c|}
\hline Year & United States* & Japan** & Europe (Big Five) ${ }^{* * * *}$ & Total World \\
\hline \multicolumn{5}{|l|}{$1973 t$} \\
\hline 1 & & & & 2,600 \\
\hline$\|$ & & & & 2,900 \\
\hline$\| 11$ & 1,058 & 322 & & 3,200 \\
\hline IV & & & & 3,100 \\
\hline \multicolumn{5}{|l|}{$1974 \dagger$} \\
\hline 1 & 995 & 277 & & 3,000 \\
\hline$\|$ & 1,102 & 345 & & 3,400 \\
\hline III & 1,156 & 381 & & 3,700 \\
\hline IV & 1,115 & 356 & & 3,600 \\
\hline \multicolumn{5}{|l|}{1975} \\
\hline 1 & 1,076 & 314 & & 3,300 \\
\hline$\|$ & 1,071 & 335 & & 3,400 \\
\hline III & 1,147 & 354 & & 3,600 \\
\hline IV & 1,111 & 348 & & 3,500 \\
\hline \multicolumn{5}{|l|}{1976} \\
\hline 1 & 1,060 & 314 & 733 & 3,200 \\
\hline$\|$ & 1,108 & 347 & 783 & 3,500 \\
\hline III & 1,191 & 387 & 868 & 3,700 \\
\hline IV & 1,111 & 377 & 860 & 3,600 \\
\hline \multicolumn{5}{|l|}{1977} \\
\hline 1 & 1,086 & 349 & 813 & 3,400 \\
\hline$\|$ & 1,195 & 385 & 854 & 3,700 \\
\hline III & 1,303 & 402 & 909 & 4,000 \\
\hline IV & 1,311 & 407 & 894 & 4,000 \\
\hline \multicolumn{5}{|l|}{1978} \\
\hline 1 & 1,167 & 383 & 790 & 3,500 \\
\hline$\|$ & 1,185 & 396 & 809 & 3,600 \\
\hline III & 1,261 & 392 & 838 & $3,800 t$ \\
\hline IV & 1,324 & 411 & 808 & $3,794 t$ \\
\hline \multicolumn{5}{|l|}{1979} \\
\hline 1 & 1,170 & 380 & 766 & $3,427 \dagger$ \\
\hline 11 & 1,210 & 392 & 831 & $3,700 t$ \\
\hline III & 1,308 & 434 & 963 & \\
\hline
\end{tabular}

•US stocks include Strategic Petroleum Reserves which held about 91.7 million bbl as of October 31, 1979.

- "Japanese stocks include government-owned strategic stockpiles. The amount was 33 million bbl at the end of June 1979 -."Europe's "Big Five" include Germany, France, UK, Italy and the Benelux couatries.

tEstimated 
Oil Stocks: Free World, U.S., Japan and Europe, 1973 -

End of Quarter

(In Billions of Barrels)

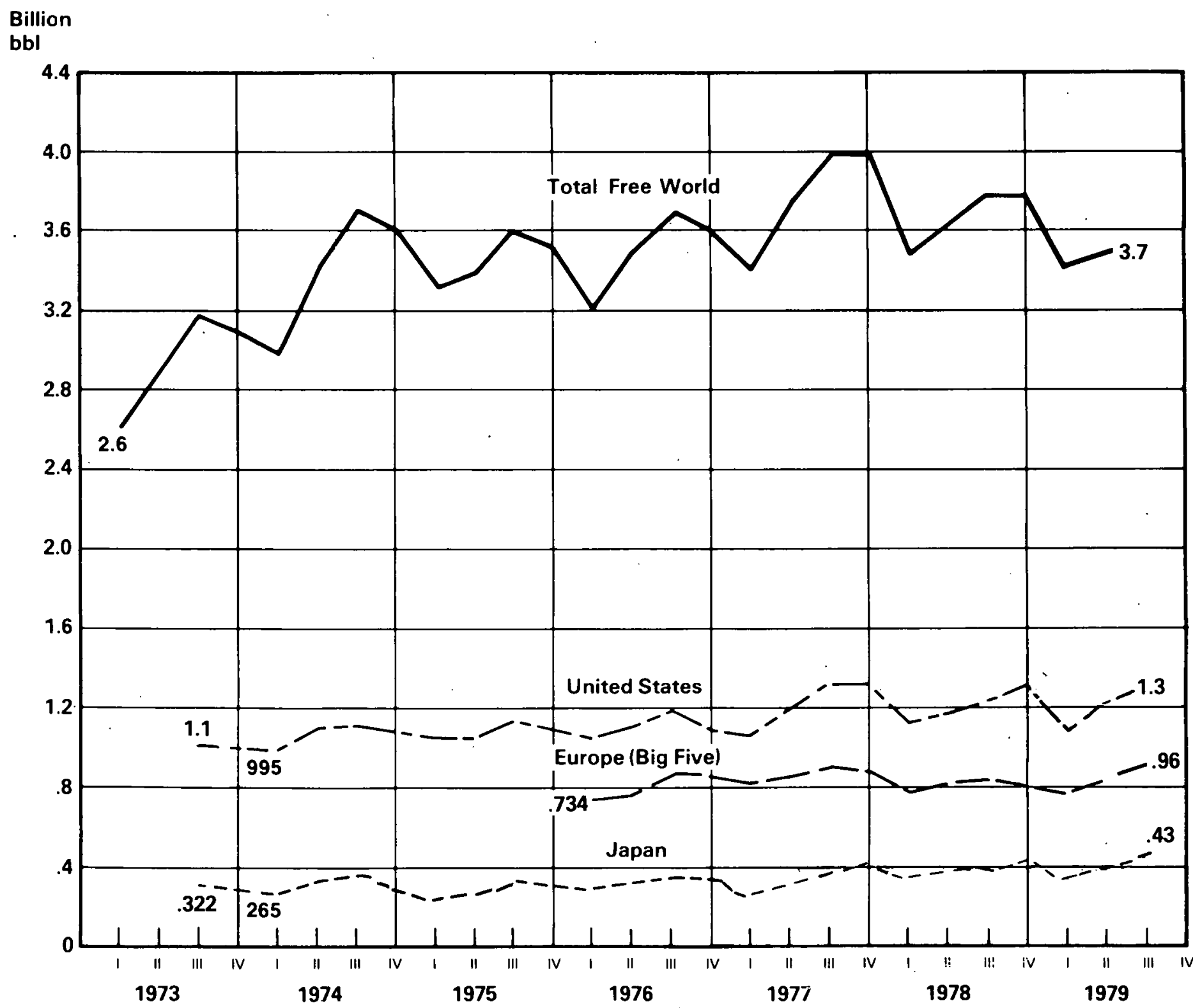

Source: CIA, Energy Branch; OECD. 
Petroleum Consumption by Industrial Countries, 1973 (In Thousands of Barrels a Day)

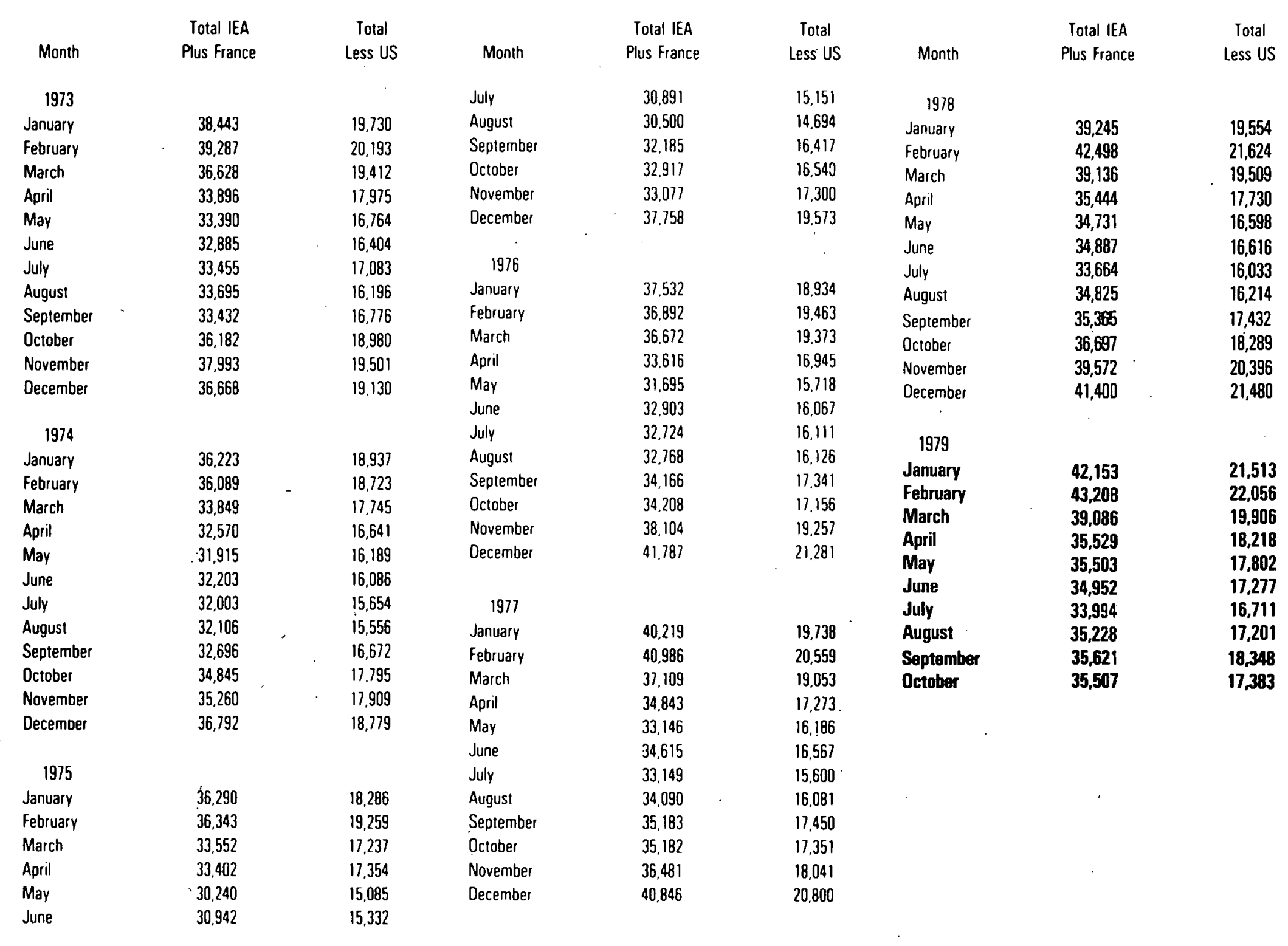

March 1980 


\section{Petroleum Consumption by Industrial Countries, 1973 - (In Millions of Barrels a Day)}

\section{MMBD}

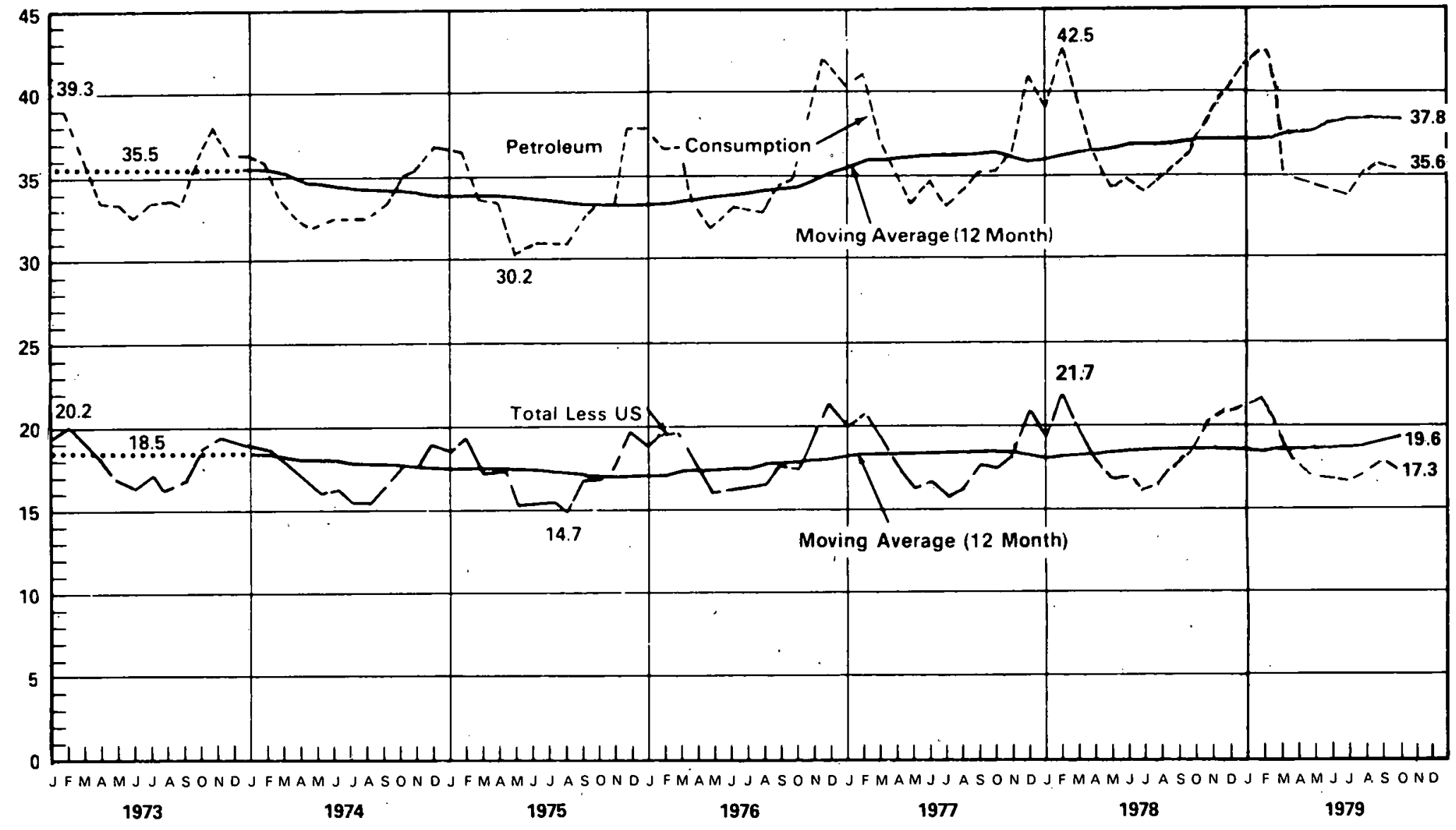

- Consumption is that of the 20 IEA countries plus France.

Source: CIA Energy Branch

Australia joined the IEA in March 1979.

- Except for the U.S.,consumption excludes bunkers, refinery

fuel and losses. 
USSR Crude Oil Production and Exports, 1974 -

(In Thousands of Barrels a Day)

\begin{tabular}{|c|c|c|}
\hline i & & $\begin{array}{l}\text { Annual } \\
\text { Exports }\end{array}$ \\
\hline $\begin{array}{l}\text { Month } \\
1974\end{array}$ & 1974 & (Total) \\
\hline January & 8,950 & \\
\hline February & 8,840 & \\
\hline March & 9,020 & \\
\hline April & 9,030 & \\
\hline May & 9,140 & \\
\hline June & 9,170 & \\
\hline July & 9,210 & \\
\hline August & 9,040 & \\
\hline September & 9,390 & \\
\hline October & 9,400 & \\
\hline November & 9,440 & \\
\hline December & 9,440 & \\
\hline AVERAGE & 9,180 & 2,340 \\
\hline \multicolumn{3}{|l|}{1975} \\
\hline January & 9,490 & \\
\hline February & 9,590 & \\
\hline March & 9,680 & \\
\hline April & 9,680 & \\
\hline May & 9,730 & \\
\hline June & 9,810 & \\
\hline July & 9,800 & \\
\hline August & 9,890 & \\
\hline September & 9,970 & \\
\hline October & 10,030 & \\
\hline November & 10,020 & \\
\hline December & 10,080 & \\
\hline AVERAGE & 9,820 & 2,600 \\
\hline \multicolumn{3}{|l|}{1976} \\
\hline January & 10,030 & \\
\hline February & 10,170 & \\
\hline March & 10,270 & \\
\hline April & 10,240 & \\
\hline
\end{tabular}

\begin{tabular}{ll} 
& \\
Month & Production \\
May & \\
June & 10,240 \\
July & 10,320 \\
August & 10,380 \\
September & 10,410 \\
October & 10,460 \\
November & 10,550 \\
December & 10,640 \\
AVERAGE & 10,670 \\
1977 & 10,390 \\
January & \\
February & 10,640 \\
March & 10,690 \\
April & 10,830 \\
May & 10,850 \\
June & 10,860 \\
July & 10,930 \\
August & 10,950 \\
September & 10,970 \\
October & 11,000 \\
November & 11,090 \\
December & 11,050 \\
AVERAGE & 11,110 \\
1978 & 10,920 \\
January & \\
February & 11,110 \\
March & 11,180 \\
April & 11,280 \\
May & 11,310 \\
June & 11,350 \\
July & 11,390 \\
August & 11,440 \\
& 11,490 \\
& \\
\hline &
\end{tabular}

Annual

Exports

(Total)

Month

September

October

November

December

AVERAGE

1979

January

February

March

April

May

June

July

August

September

October

November

December

AVERAGE

1980

January
Annual

Exports

(Total)

3,160

11,600

11,600

11,600

11,730

11,350

11,680

11,630

11,800

11,680

11,870

11,920

$11,960^{\circ}$

$11,710^{*}$

$3,000^{\circ}$

11,870

11,860 
USSR Crude Oil Production, 1975 -

(Including Natural Gas Liquids)

Rate of Change in Production Over

Same Period of Preceding Year

\section{Percent}

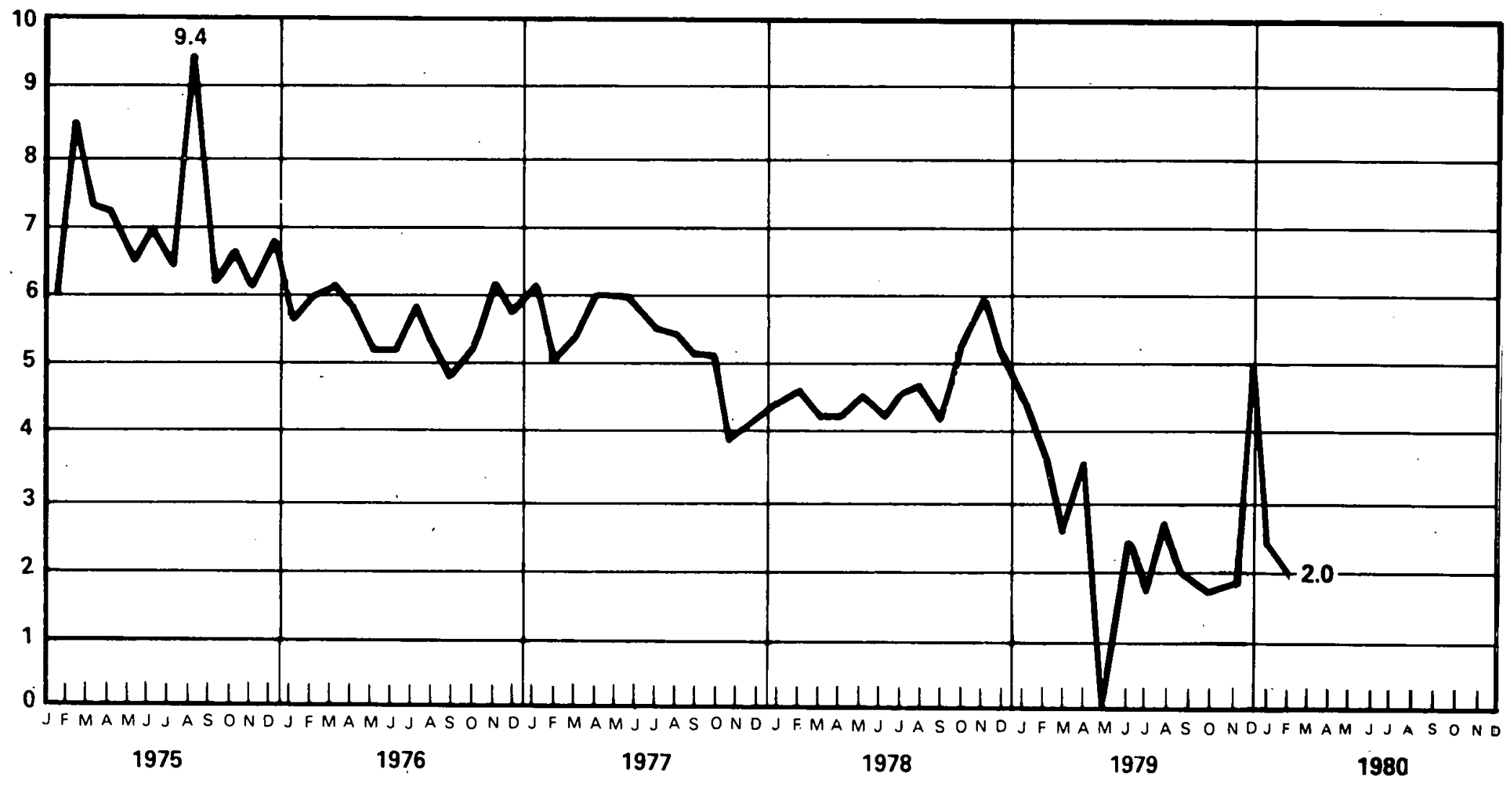


Free World and U.S. Nuclear Generation Capacity, 1973 (In Megawatts).

\begin{tabular}{|c|c|c|c|c|c|c|c|c|c|c|c|}
\hline Month & $\begin{array}{l}\text { Free World } \\
\text { Ex.US }\end{array}$ & $\begin{array}{l}\text { United } \\
\text { States }\end{array}$ & $\begin{array}{c}\text { Total } \\
\text { Free World }\end{array}$ & Month & $\begin{array}{c}\text { Free World } \\
\text { Ex-US }\end{array}$ & $\begin{array}{l}\text { United } \\
\text { States }\end{array}$ & $\begin{array}{c}\text { Total } \\
\text { Free World }\end{array}$ & Month & $\begin{array}{l}\text { Free World } \\
\text { Ex.US }\end{array}$ & $\begin{array}{l}\text { United } \\
\text { States }\end{array}$ & $\begin{array}{c}\text { Total } \\
\text { Free World }\end{array}$ \\
\hline 1973 & & & & July & 27,064 & 37,334 & 64,398 & 1978 & & & \\
\hline January & 18,109 & 15,449 & 33,558 & August & 27,984 & 37,330 & 65,314 & January & 43,353 & 49,250 & 92,603 \\
\hline February & 18,759 & 15,471 & 34,230 & September & 28,394 & 37,330 & 65,724 & February & 43,330 & 49,300 & 92,630 \\
\hline March & 18,779 & 15,471 & 34,250 & October & 28,953 & 38,130 & 67,083 & March & 43,350 & 50,430 & 93,780 \\
\hline April & 18,779 & 16,295 & 35,074 & November & 29,779 & 36,570 & 66,349 & April & 44,140 & 50,430 & 94,570 \\
\hline May & 19,237 & 16,295 & 35,532 & December & 29.792 & 39,399 & 69,191 & May & 45,100 & 50,470 & 95,570 \\
\hline June & 19,237 & 17,023 & 36,260 & & & & & June & 45,100 & 51,400 & 96,500 \\
\hline July & 19,237 & 17,023 & 36,260 & 1976 & & & & July & 45,130 & 51,400 & 96,530 \\
\hline August & 19,237 & 17,023 & 36,260 & January & 29,792 & 39,400 & 69,192 & August & 46,570 & 51,400 & 97,970 \\
\hline September & 19,237 & 17,910 & 37,147 & February & 29,770 & 39,350 & 69,120 & September & 46,570 & 52,360 & 98,930 \\
\hline October & 19,237 & 19,417 & 38,654 & March & 31,094 & 39,350 & 70,444 & October & 48,337 & 53,183 & 101,520 \\
\hline November & 19,237 & 19,417 & 38,654 & April & 31,094 & 39,370 & 70,464 & November & 51,030 & 53,140 & 104,170 \\
\hline December & 19.714 & 20,887 & 40,601 & May & 32,394 & 41,240 & 73,634 & December & 50,940 & 54,120 & 105,060 \\
\hline & & & & June & 33,054 & 42,085 & 75,139 & & & & \\
\hline 1974 & & & & July & 33,057 & 42.715 & 75,782 & 1979 & & & \\
\hline January & 19,972 & 22,810 & 42,782 & August & 33,067 & 45,680 & 78,747 & January & 52,360 & 54,180 & 106,540 \\
\hline February & 19,976 & 23,648 & 43,624 & September & 34,729 & 43,200 & 77,929 & February & 53,300 & 54,180 . & 107,480 \\
\hline March & 20,436 & 24,748 & 45,184 & October & 35,479 & 43,200 & 78,679 & March & 55,150 & 54,180 & 109,330 \\
\hline April & 20,436 & 25,208 & 45,644 & November & 36,965 & 43,200 & 80,165 & April & 56,230 & 54,180 & 110,410 \\
\hline May & 20,428 & 25,991 & 46,419 & December & 36,965 & 44,080 & 81,045 & May & 56,200 & 54,180 & 110,380 \\
\hline June & 20,416 & 26,016 & 46,432 & & & & & June & 56,200 & 54.180 & 110,380 \\
\hline July & 21,420 & 26,551 & 47,971 & 1977 & & & & July & 56,190 & 54,220 & 110,410 \\
\hline August & 22,580 & 28,293 . & 50,873 & January & 37,815 & 44,080 & 81,895 & August & 57,160 & 54,200 & ' 111,360 \\
\hline September & 22,580 & 29,180 & 51,760 & February & 37,815 & 44,910 & 82,725 & September & 58,470 & 54,200 & 112,670 \\
\hline October & 23,940 & 32,340 & 56,280 & March & 38,758 & 44,910 & 83,668 & October & 59,559 & 54,181 & 113,741 \\
\hline November & 24,667 & 32,827 & 57,494 & April & 40,304 & 46,090 & 86,394 & November & 61,703 & 54,193 & $115,896$. \\
\hline December & 25,136 & 33,338 & 58,474 & May & 40,381 & 46,040 & 86,421 & December & 61,703 & 54,191 & 115,894 \\
\hline & & & & June & 40,381 & 46,230 & 86,611 & 1980 & & & \\
\hline 1975 & & & & July & 40,361 & 47.110 & 87,471 & January & 61,452 & 54,191 & 115,643 \\
\hline January & 26,454 & 34,626 & 61.080 & August & 40,301 & 46,930 & 87,231 & & & & \\
\hline February & 26,936 & 35,429 & 62,365 & September & 40,957 & 47,073 & 88,030 & & & & \\
\hline March & 26,569 & 35,446 & 62,015 & October & 41,858 & 47,311 & 89,169 & & & & \\
\hline April & 26,449 & 35,495 & 61,944 & November & 42,492 & 47,370 & 89,862 & & & & . \\
\hline May & 27,064 & 36,495 & 63,559 & December & 43,352 & 48,340 & 91,692 & & & & \\
\hline June & 27,064 & 37,344 & 64,408 & \multicolumn{4}{|c|}{18} & & & \multicolumn{2}{|c|}{ March 1980} \\
\hline
\end{tabular}




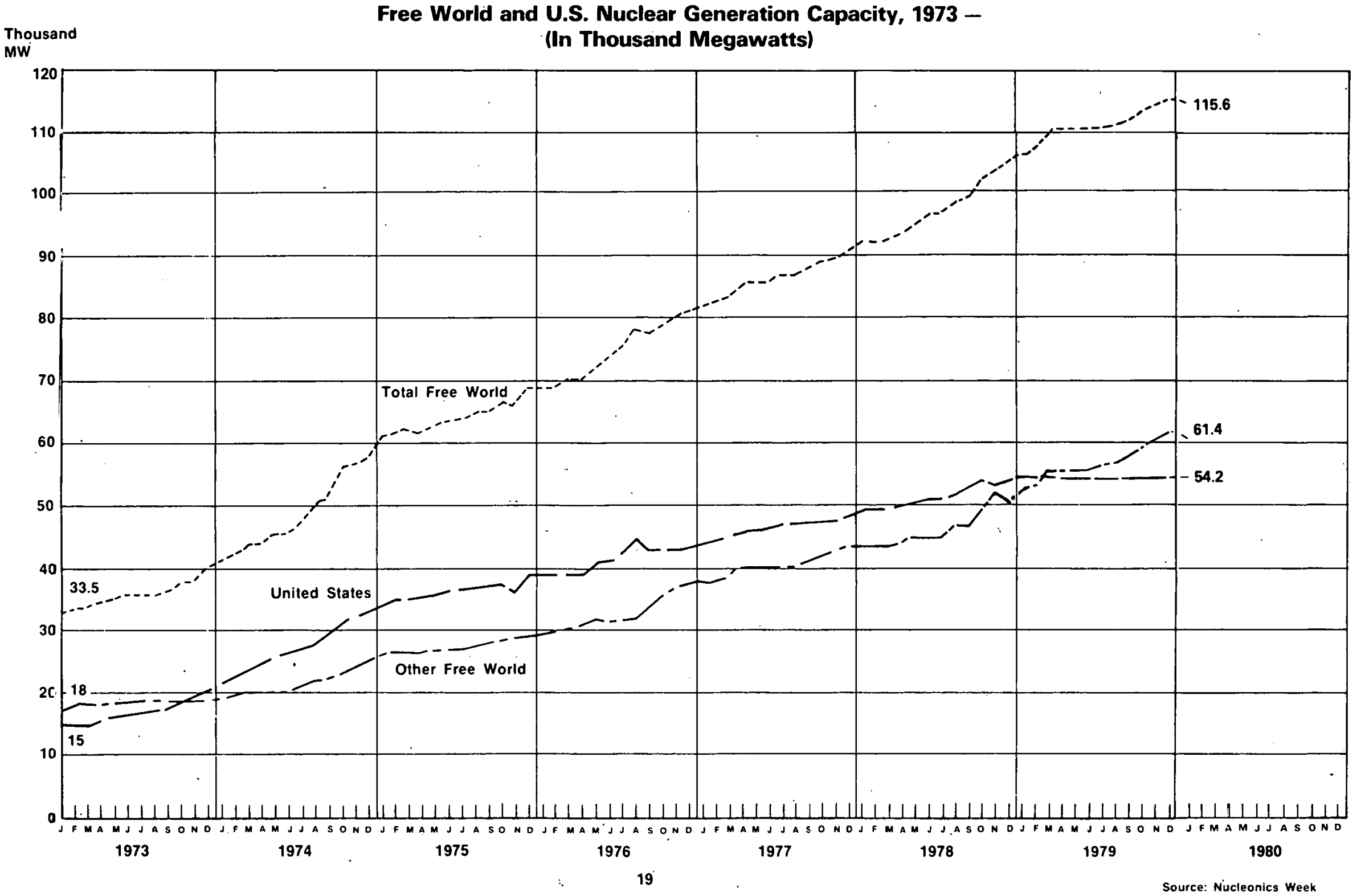

March 1980 
U.S. Imports of Crude Oil and Products, 1973 (In Thousands of Barrels a Day)

\begin{tabular}{|c|c|c|c|c|c|c|c|c|c|c|c|}
\hline & Crude & & & & Crude & & & & Crude & & \\
\hline Month & Oil & Product & Total & Month & Oil & Product & Total & Month & Oil & Product & Total \\
\hline 1973 & & & & & & & & & & & \\
\hline January & 2,732 & 3,125 & 5,857 & July & 4,192 & 1,767 & 5,959 & 1978 & & & \\
\hline February & 2,873 & 3,635 & 6,508 & August & 4,581 & 1,717 & 6,298 & January & 6,126 & 2,092 & 8,218 \\
\hline March & 3,162 & 3,448 & 6,610 & September & 4,689 & 2,115 & 6,804 & February & 5,655 & 2,355 & 8,010 \\
\hline April & 3,049 & 2,545 & 5,594 & October & 4,389 & 1,940 & 6,329 & March & 6,031 & 2,338 & 8,369 \\
\hline May & 3,215 & 2,626 & 5,841 & November & 4,623 & 1,796 & 6,419 & April & 5,519 & 2,115 & 7,634 \\
\hline June & 3,220 & 2,670 & 5,890 & December & 4,476 & 1,949 & 6,425 & May & 5,594 & 1,804 & 7,398 \\
\hline July & 3,501 & 2,678 & 6,179 & AVERAGE & 4,105 & 1,951 & 6,056 & June & 6,322 & 1,640 & 7,962 \\
\hline August & 3,593 & 2,999 & 6,592 & & & & & July & 6,175 & 1,948 & 8,123 \\
\hline September & 3,471 & 2,941 & 6,412 & $\begin{array}{r}1976 \\
\text { January }\end{array}$ & 4595 & 2.071 & 6,666 & August & 6,251 & 1,858 & 8,109 \\
\hline October & 3,739 & 2,894 & 6,633 & $\begin{array}{l}\text { January } \\
\text { February }\end{array}$ & 4,208 & $\begin{array}{l}2,011 \\
2,423\end{array}$ & $\begin{array}{l}0,000 \\
6,631\end{array}$ & September & 6,829 & 1,983 & 8,811 \\
\hline November & 3,452 & 3,470 & 6,922 & March & 4,738 & 1,945 & 6,683 & October & 6,400 & 1,718 & 8,119 \\
\hline December & 2,891 & 3,164 & 6,055 & April & 4,790 & 1,805 & 6,595 & November & 6,643 & 2,021 & 8,664 \\
\hline AVERAGE & 3,244 & 3,012 & 6,256 & May & 4,669 & 1,654 & 6,323 & December & 6,751 & 2,245 & 8,996 \\
\hline 1974 & & & & June & 5,621 & 1,858 & 7,479 & AVERAGE & 6,195 & 2,008 & 8,202 \\
\hline January & 2,382 & 2,973 & 5,355 & July & 5,792 & 2,099 & 7,890 & & & & \\
\hline February & 2,248 & 2,973 & 5,221 & August & 5,556 & 1,826 & 7,382 & 1979 & & & \\
\hline March & 2,462 & 2,753 & 5,215 & September & 5,875 & 2,038 & 7,913 & January & 6,562 & 2,205 & 8,767 \\
\hline April & 3,267 & 2,703 & 5,970 & October & 5,699 & 1,808 & 7,508 & February & 6,249 & 2,069 & 8,318 \\
\hline May & 3,908 & 2,580 & 6,488 & November & 5,946 & 2,115 & 8,060 & March & 6,180 & 2,385 & 8,565 \\
\hline June & 3,925 & 2,493 & 6,418 & December & 5,925 & 2,468 & 8,393 & April & 6,047 & 1,666 & 7,713 \\
\hline July & 4,091 & 2,397 & 6,488 & AVERAGE & 5,287 & 2,026 & 7,313 & May & 6,092 & 1,809 & 7,901 \\
\hline August & 3,924 & 2.434 & 6,358 & & & & & June & 6,523 & 1,672 & 8,195 \\
\hline September & 3,797 & 2,225 & 6,022 & 1977 & & & & July & 6,120 & 1,783 & 7,902 \\
\hline October & 3,810 & 2,340 & 6,150 & January & 6,281 & 2,622 & 8,903 & August & 6,692 & 1,675 & 8,367 \\
\hline November & 3,958 & 2,704 & 6,662 & February & 6,659 & 3,338 & 9,997 & September & 6,321 & 1,599 & 7,921 \\
\hline December & 3,869 & 2,781 & 6,650 & March & 6,699 & 2,684 & 9,383 & October & 6,366 & 1,609 & 7,976 \\
\hline AVERAGE & 3,477 & 2,635 & 6,112 & April & 6,821 & 1,902 & 8,723 & November & 5,711 & 1,745 & 7,456 \\
\hline & & & & May & 6,818 & 1,753 & 8,571 & December* & 6,415 & 2,112 & 8,527 \\
\hline $\begin{array}{r}1975 \\
\text { January }\end{array}$ & & & & June & 7,065 & 1,872 & 8,937 & AVERAGE & 6,275 & 1,861 & 8,136 \\
\hline January & 4,029 & 2,832 & 6,861 & July & 7,068 & 2,027 & 9,095 & & & & \\
\hline February & 3,828 & 2,348 & 6,176 & August & 6,395 & 2,179 & 8,574 & 1980 & & & \\
\hline March & 3,656 & 2,074 & 5,730 & September & 6,429 & 2,137 & 8,567 & January* & 6,447 & 2,015 & 8,462 \\
\hline April & 3,378 & 1,662 & 5,040 & October & 6,409 & 1,862 & 8,272 & & & & \\
\hline May & 3,486 & 1,728 & 5,214 & November & 6,248 & 1,814 & 8,062 & Note: 1978 a & fures re & Inthly Energ & \\
\hline June & 3,905 & 1,502 & 5,406 & December & 6,248 & 2,198 & 8,446 & *API Prelimir & & & \\
\hline & & & & AVERAGE & 6,594 & 2,193 & 8,787 & & & & \\
\hline & & & & & & 0 & & & & $\mathbf{M z}$ & \\
\hline
\end{tabular}


U.S. Imports of Crude Oil and Products, 1973 -

(In Millions of Barrels a Day)

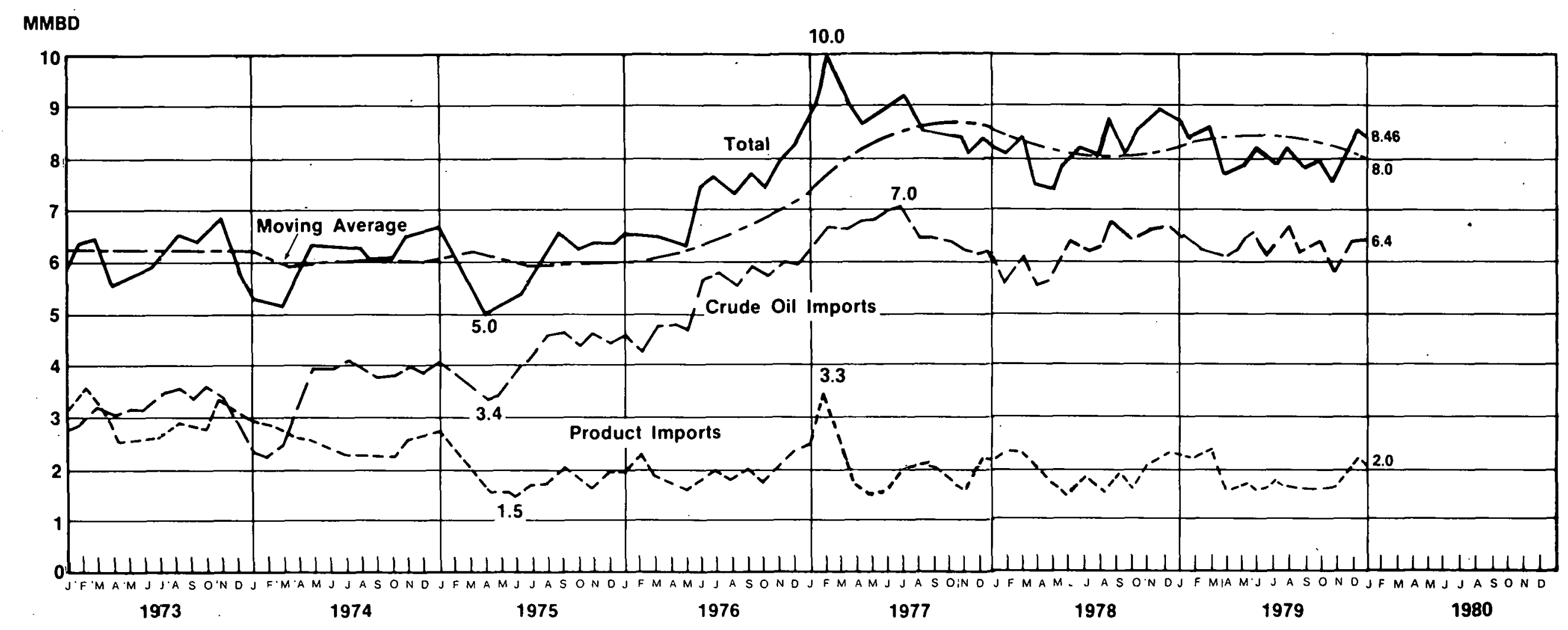

- Moving awerage is last 12 months.

- SPR purchases not included.

Source: Bureau of Mines, DOE/EIA, API 
Landed Cost of Saudi Crude Oil in Current and 1974 Dollars

(In Dollars per Barrel)

\begin{tabular}{|c|c|c|c|c|c|c|c|c|}
\hline Month & $\begin{array}{l}\text { Landed Cost of } \\
\text { Saudi Crude } \\
\text { (Actual) }\end{array}$ & $\begin{array}{l}\text { Cost in } \\
1974 \text { Dollars } \\
\text { (CPI Deflator) }\end{array}$ & Month & $\begin{array}{l}\text { Landed Cost of } \\
\text { Saudi Crude } \\
\text { (Actuall) }\end{array}$ & $\begin{array}{l}\text { Cost in } \\
1974 \text { Dollars } \\
\text { (CPI Deflator) }\end{array}$ & Month & $\begin{array}{l}\text { Landed Cost of } \\
\text { Saudi Crude } \\
\text { (Actual) }\end{array}$ & $\begin{array}{l}\text { Cost in } \\
1974 \text { Dollars } \\
\text { (CPI Deflator) }\end{array}$ \\
\hline 1974 & & & 1976 & & & 1978 & & \\
\hline April & 11.59 & 11.90 & July & 13.02 & 11.24 & October & 14.07 & 10.28 \\
\hline May & 11.53 & 11.70 & August & 13.03 & 11.19 & November & 14.07 & 10.34 \\
\hline June & 11.32 & 11.38 & September & 12.87 & 11.02 & December & 14.00 & 10.11 \\
\hline July & 11.97 & 11.95 & October & 12.87 & 10.97 & & & \\
\hline August & 12.16 & 11.98 & November & 13.01 & 11.06 & 1979 & & \\
\hline September & 11.45 & 11.15 & December & 13.02 & 11.03 & January & 14.73 & 10.64 \\
\hline October & 11.51 & 11.11 & & & & February & 14.88 & 10.61 \\
\hline November & 12.15 & 11.63 & 1977 & & & March & 15.28 & 10.79 \\
\hline \multirow[t]{2}{*}{ December } & 11.75 & 11.16 & January & 13.22 & 11.14 & April & 16.18 & 11.29 \\
\hline & & & February & 13.32 & 11.11 & May & 16.29 & 11.24 \\
\hline 1975 & & & March & 13.50 & 11.19 & June & 17.98 & 12.26 \\
\hline January & 12.07 & 11.42 & April & 13.41 & 11.02 & July & 20.06 & 13.54 \\
\hline February & 11.94 & 11.22 & May & 13.49 & 11.03 & August & 19.85 & 13.26 \\
\hline March & 11.78 & 11.03 & June & 13.39 & 10.87 & September & 20.36 & 13.44 \\
\hline April & 12.16 & 11.32 & July & 13.64 & 11.03 & October & 22.99 & 15.06 \\
\hline May & 12.27 & 11.37 & August & 13.72 & 11.06 & Droliming & & \\
\hline June & 11.93 & 10.98 & September & 14.01 & 11.25 & & & \\
\hline July & 12.08 & 10.99 & October & 13.85 & 11.09 & & & \\
\hline August & 12.10 & 10.97 & November & 13.94 & 11.11 & & & \\
\hline September & 12.17 & 10.99 & December & 13.99 & 11.11 & & & \\
\hline October & 12.64 & 11.34 & & & & & & \\
\hline November & 12.58 & 11.22 & 1978 & & & & & \\
\hline \multirow[t]{2}{*}{ December } & 12.93 & 11.48 & January & 13.93 & 10.99 & & & \\
\hline & & & February & 13.96 & 10.94 & & & \\
\hline 1976 & & & March & 14.07 & 10.96 & & & \\
\hline January & 13.18 & 11.68 & April & 13.85 & 10.69 & & & \\
\hline February & 13.21 & 11.68 & May & 13.86 & 10.59 & & & \\
\hline March & 13.18 & 11.62 & June & 13.86 & 10.43 & & & \\
\hline April & 13.11 & 11.51 & July & 14.00 & 10.51 & & & \\
\hline May & 13.05 & 11.39 & August & 14.05 & 10.50 & & & \\
\hline June & 13.14 & 11.41 & September & 14.21. & 10.53 & & & \\
\hline
\end{tabular}




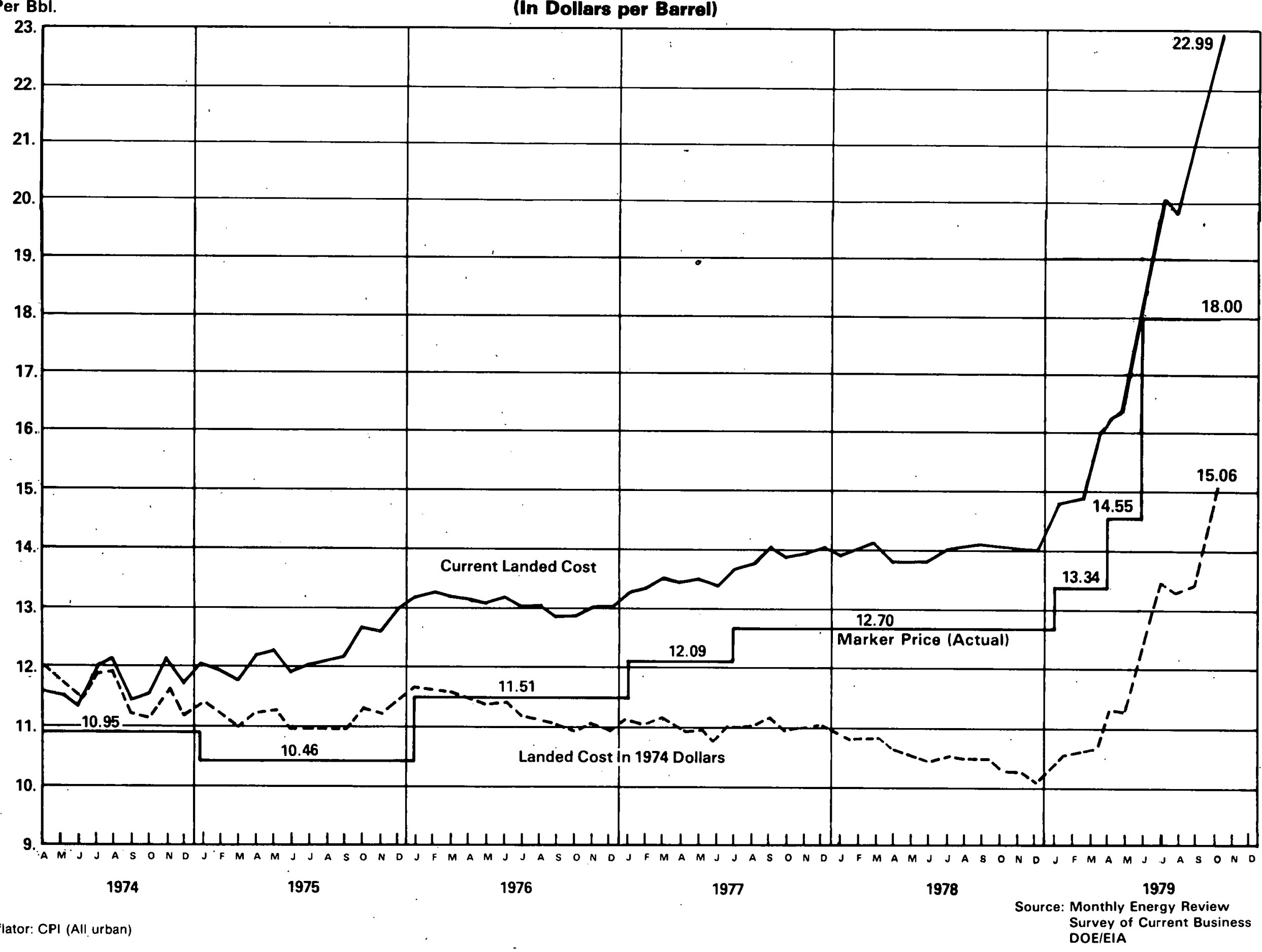


U.S. Trade in Coal, 1973 -

(In Thousand Short Tons per Month)

\begin{tabular}{|c|c|c|c|c|c|c|c|c|}
\hline 1973 & Exports & Imports & 1975 & Exports & Imports & 1978 & Exports & Imports \\
\hline January & 2,954 & 34 & September & 4,529 & 50 & January & 870 & 139 \\
\hline February & 2,669 & 31 & October & 4,647 & 27 & February. & 555 & 159 \\
\hline March & 3,377 & 36 & November & 7,593 & 112 & March & 325 & 231 \\
\hline April & 5,063 & 40 & December & 4,534 & 182 & April & 2,594 & 417 \\
\hline May & 5,140 & 125 & & & & May & 4,411 & 323 \\
\hline June & 4,969 & 102 & 1976 & & & June & 5,398 & 291 \\
\hline July . & 4,188 & 76 & & & & July & 3,531 & 313 \\
\hline August & 5,133 & 96 & Januâny & 3,697 & 118 & August & 3,568 & 227 \\
\hline September & 3,424 & 187 & February & 3,050 & 139 & September & 3,338 & 196 \\
\hline October & 5,882 & 218 & March & 3,979 & 54 & October & 4,911 & 371 \\
\hline November & 5,214 & 153 & April & 5,780 & 156 & November & 5,930 & 98 \\
\hline \multirow[t]{2}{*}{ December } & 4,889 & 115 & May & 5,667 & 116 & December & 4,394 & 188 \\
\hline & & & June & 6,569 & 265 & & & \\
\hline \multirow[t]{2}{*}{1974} & & & July & 4,880 & 134 & & & \\
\hline & & & August & 4,223 & 361 & 1979 & & \\
\hline January & 2,813 & 333 & September & 5,613 & 261 & & & \\
\hline February & 4,627 & 344 & October & 5,871 & 413 & January & & 186 \\
\hline March & 3,179 & 346 & November & 5,451 & 291 & Jaduarly & 3,526 & 186 \\
\hline April & 4,944 & 424 & December & 4,625 & 225 & February & 2,691 & .252 \\
\hline May & 6,032 & 437 & & & & March & 4,592 & 123 \\
\hline June & 6,369 & 387 & 1977 & & & April & 5,227 & 161 \\
\hline July. & 5,307 & 402 & & & & May & 6,091 & 112 \\
\hline August & 5,088 & 780 & January & 2,143 & 149 & June & 5,895 & 209 \\
\hline September & 4,893 & 436 & February & 3,079 & 130 & July & 6,249 & 88 \\
\hline October & 7,342 & 731 & March & 3,390 & 67 & August & 6,089 & 320 \\
\hline November & 6,744 & 634 & April & 5,637 & 200 & September & 5,019 & 180 \\
\hline \multirow[t]{2}{*}{ December } & 2,586 & 415 & May & 5,673 & 203 & October & 7,315 & 34 \\
\hline & & & June & 6,019 & 257 & November & 6,017 & 130 \\
\hline \multirow[t]{2}{*}{1975} & & & July & 5,158 & 304 & December & 6,072 & 146 \\
\hline & & & August & 4,279 & 274 & & & \\
\hline January & 4,254 & 540 & September & 5,037 & 458 & & & \\
\hline February & 4,470 & 606 & October & 4,871 & 595 & & & \\
\hline March & 5,653 & 411 & November & 4,491 & 503 & & & \\
\hline April & 6,159 & 192 & December & 3,910 & 514 & & & \\
\hline May & 7,011 & 334 & & & & & & \\
\hline June & 6,269 & 110 & & & & & & \\
\hline July & 4,691 & 65 & & & & & & \\
\hline August & 5,859 & 144 & & & & & & \\
\hline
\end{tabular}

Source: DOE/Bureau of Mines 
086L YOد8W

$6 \angle 6 L$

8L6L

$\angle \angle 6 L$

9L6L

SL6L

tL6L

EL6L

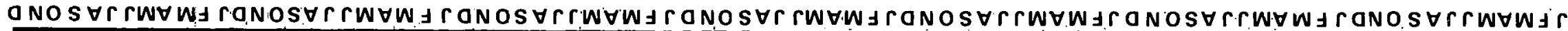

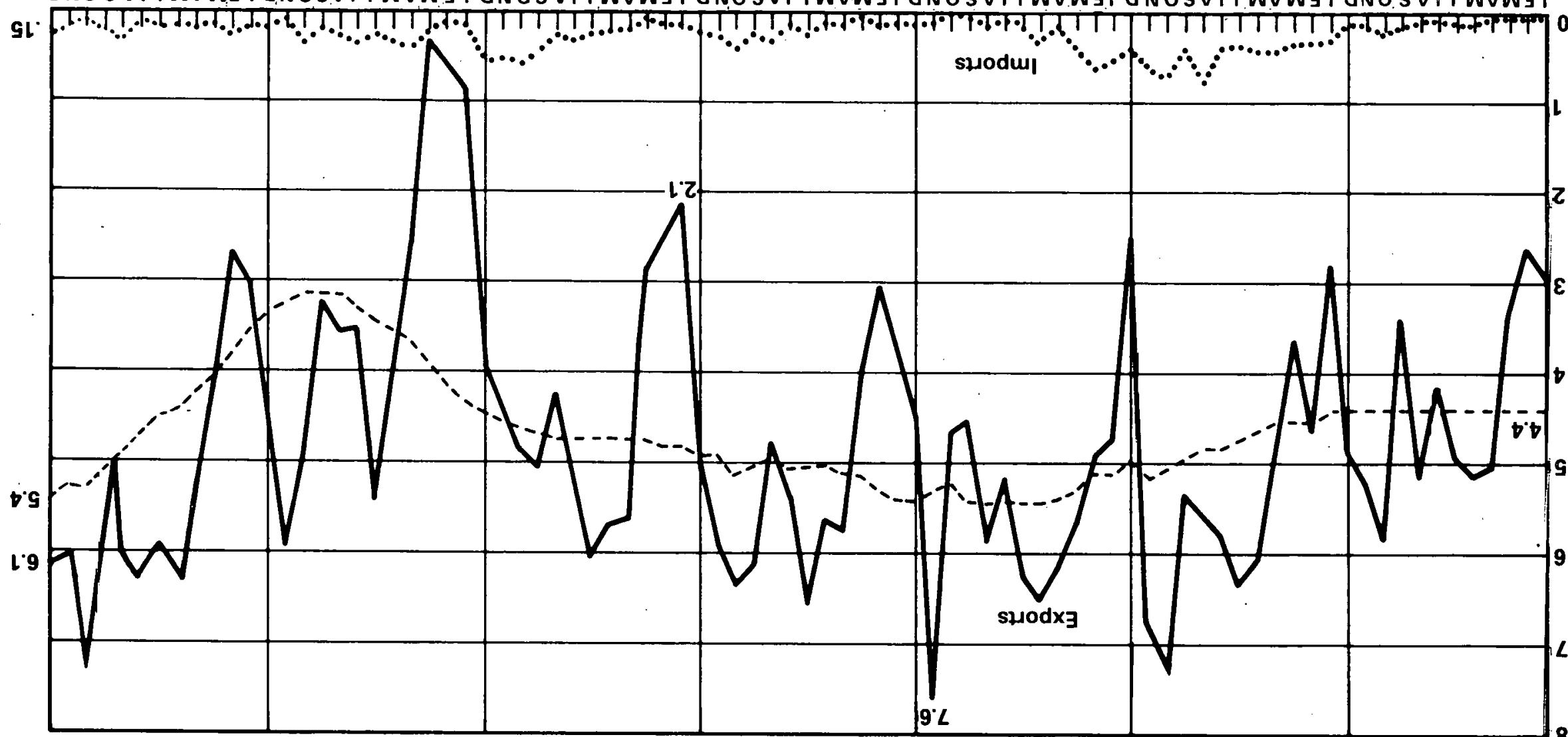


Summary of U.S. Merchandise Trade, 1976 (In Millions of Dollars, Adjusted)

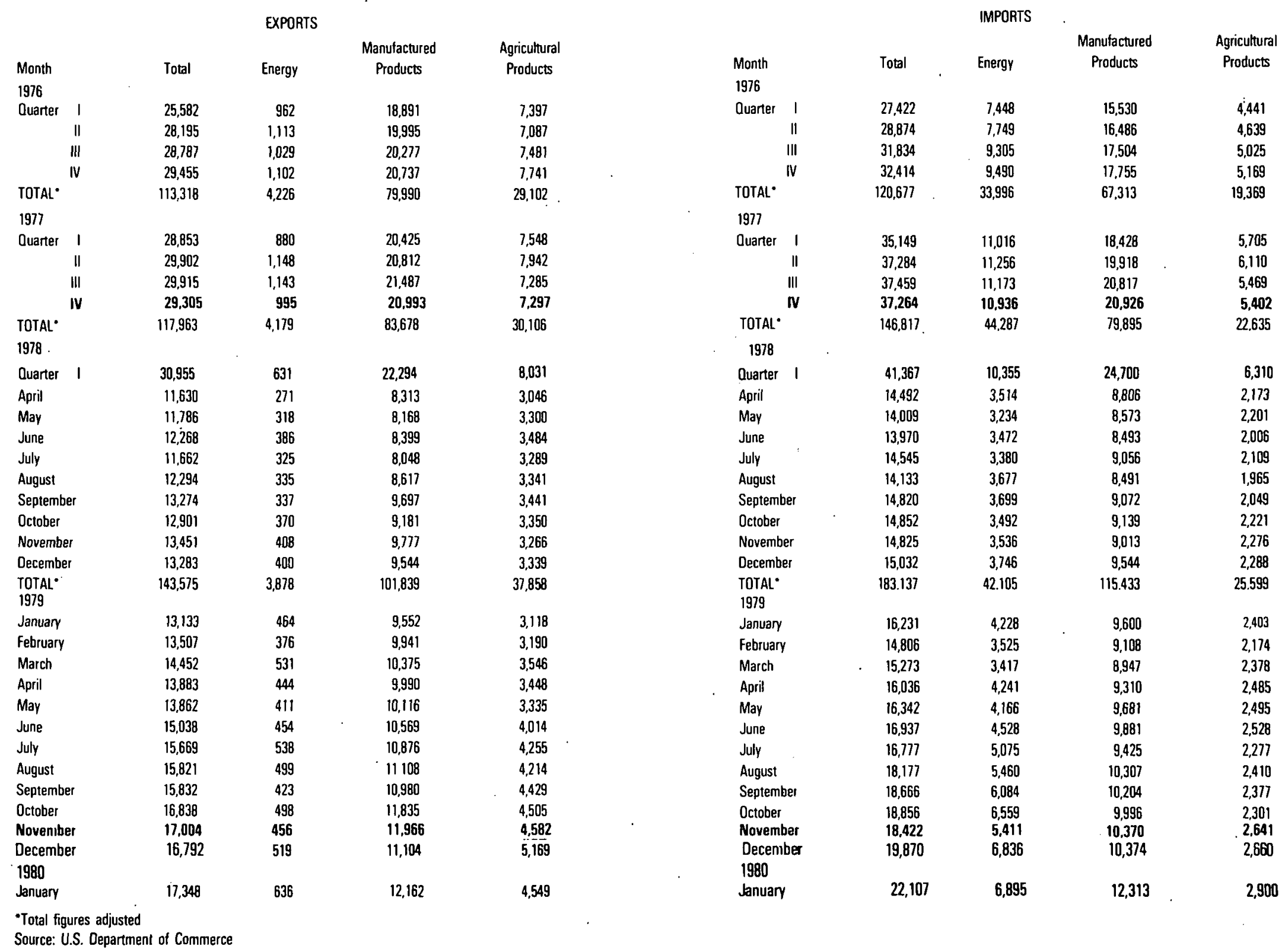


Summary of U.S. Merchandise Trade, 1976 -

Billions of

Dollars

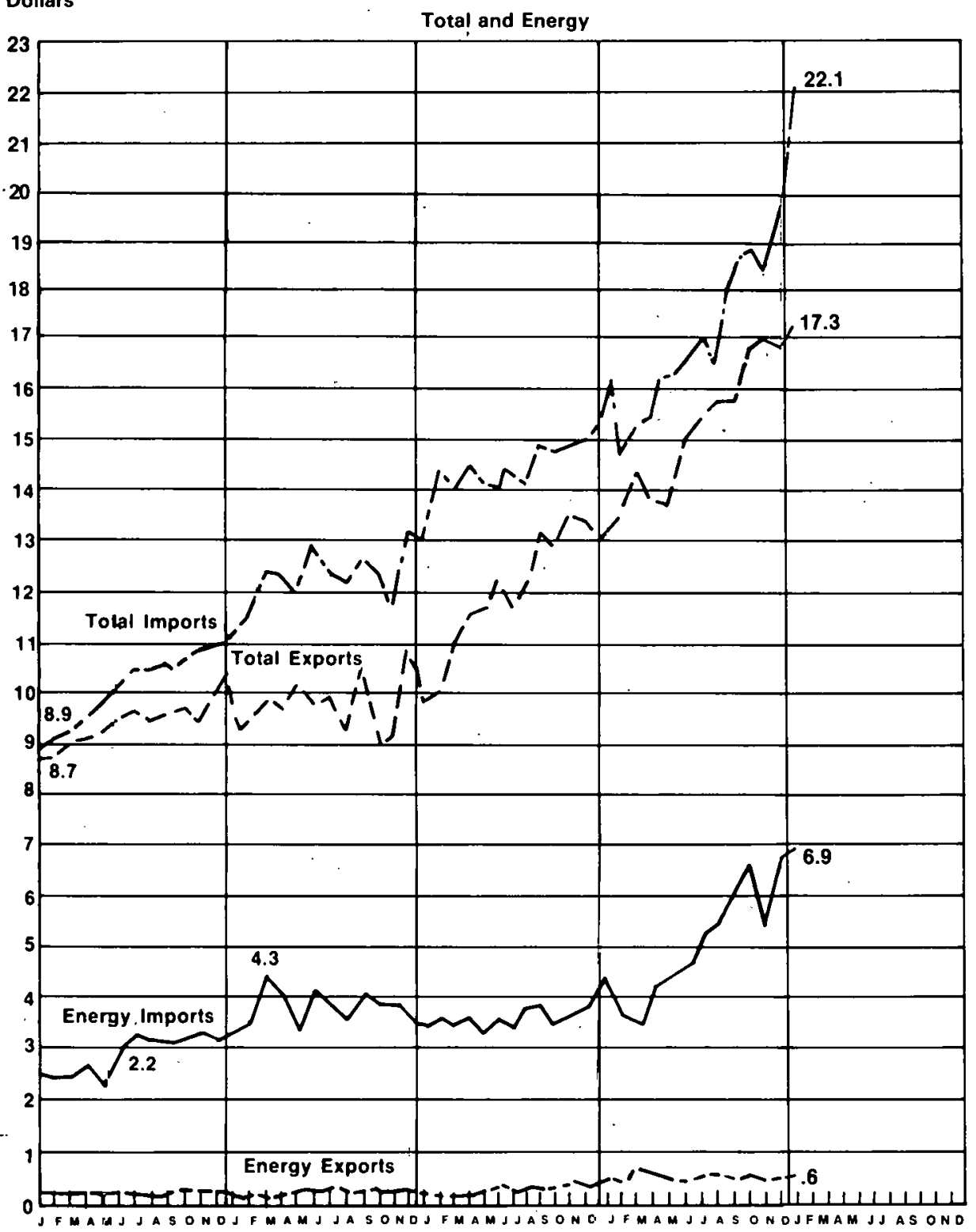

1976
1977.
1978
1979
1980

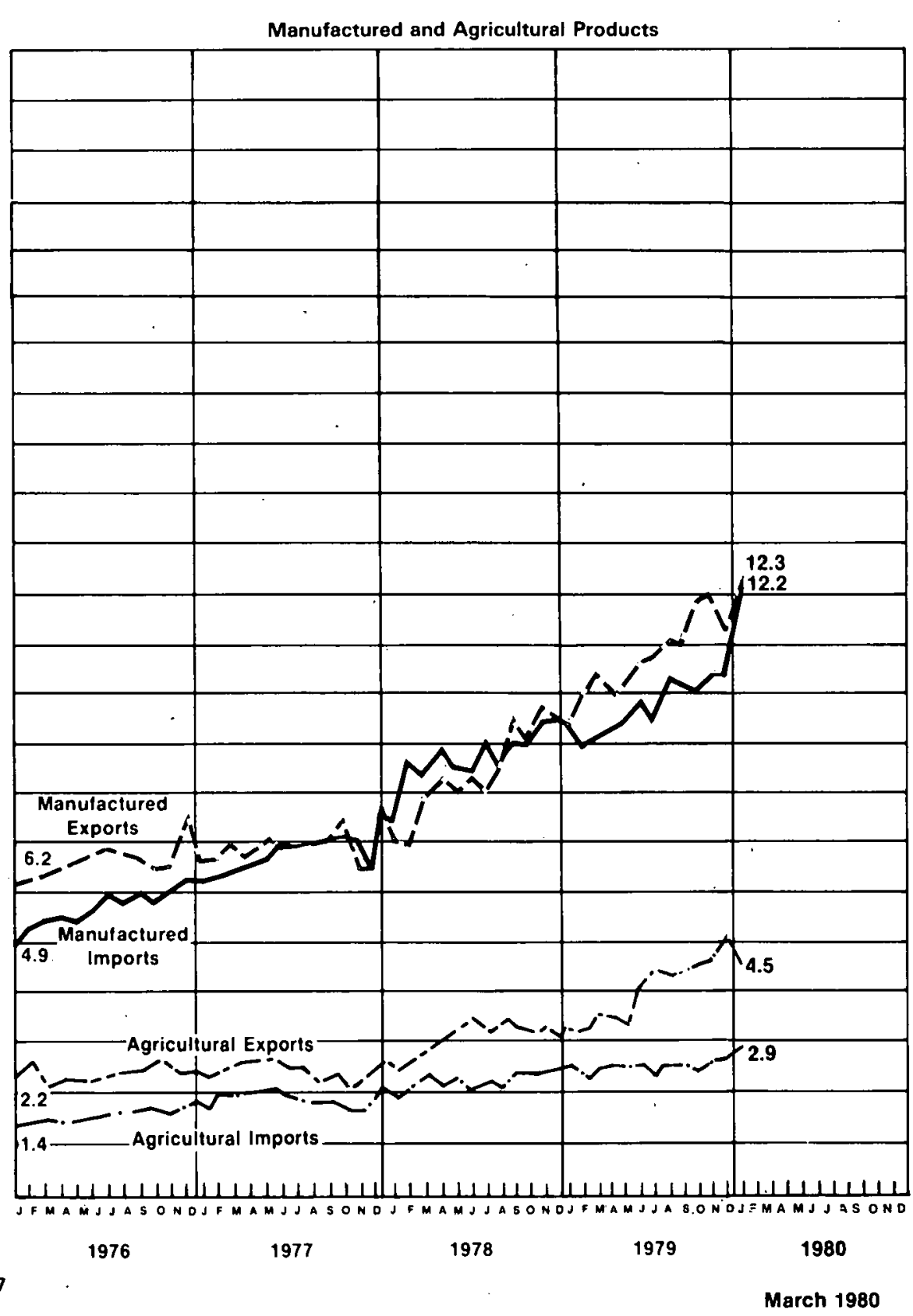


U.S. Energy/GNP Ratio

(In 1972 Dollars)

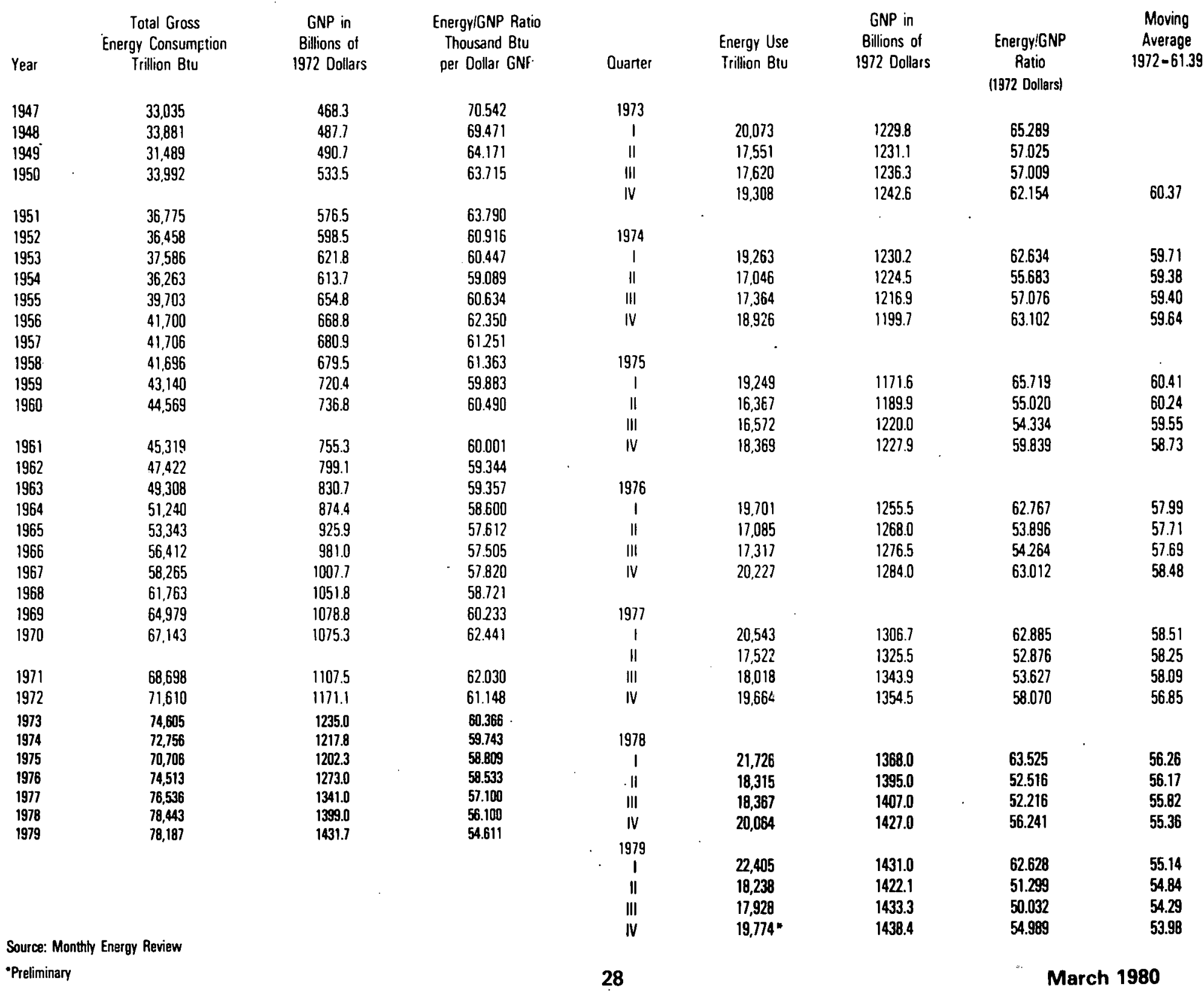




\section{U.S. Energy/GNP Ratio, 1972 Dollars}

1,000 Btu

per Dollar GNP

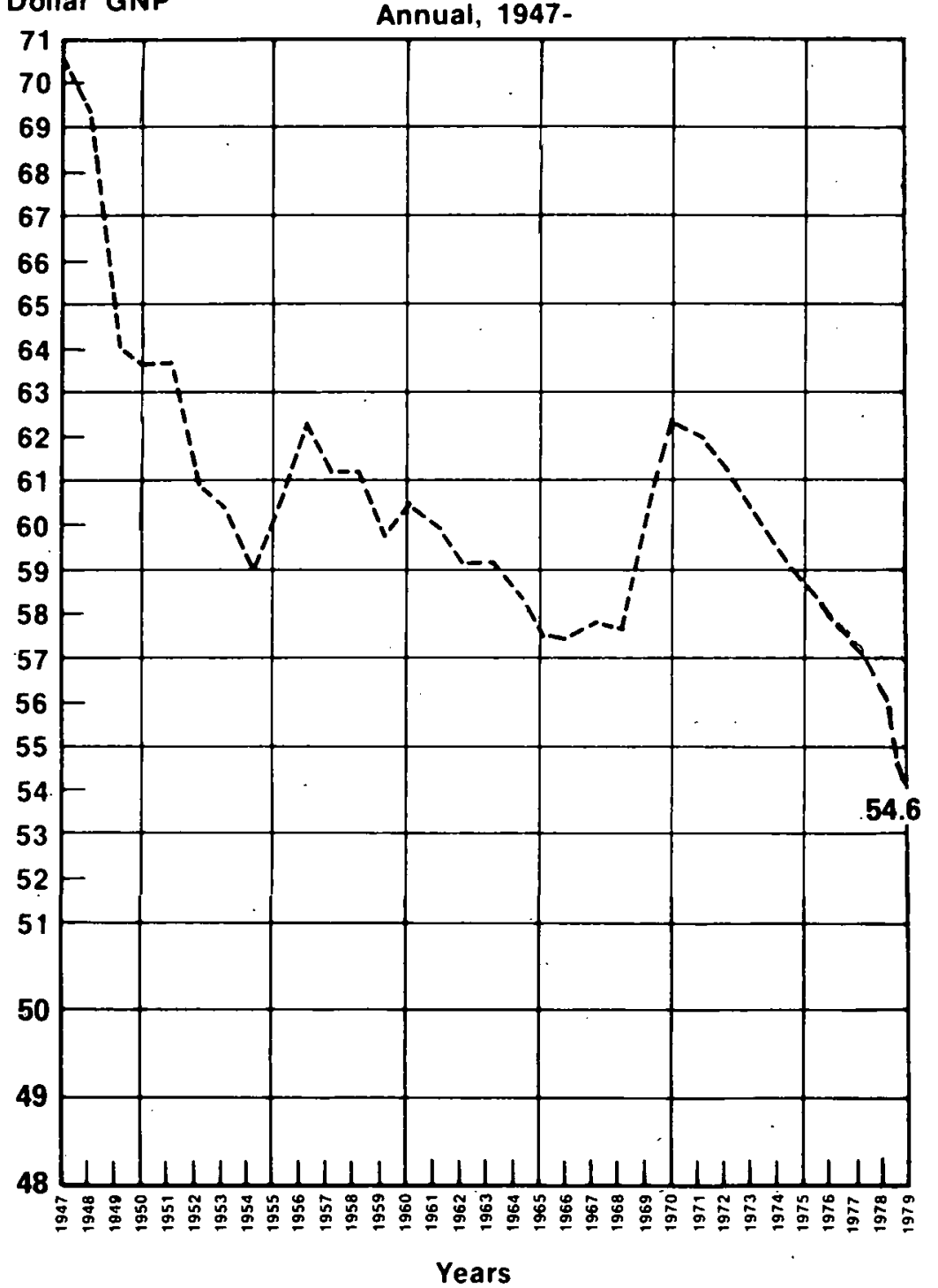

Quarterly, 1973-

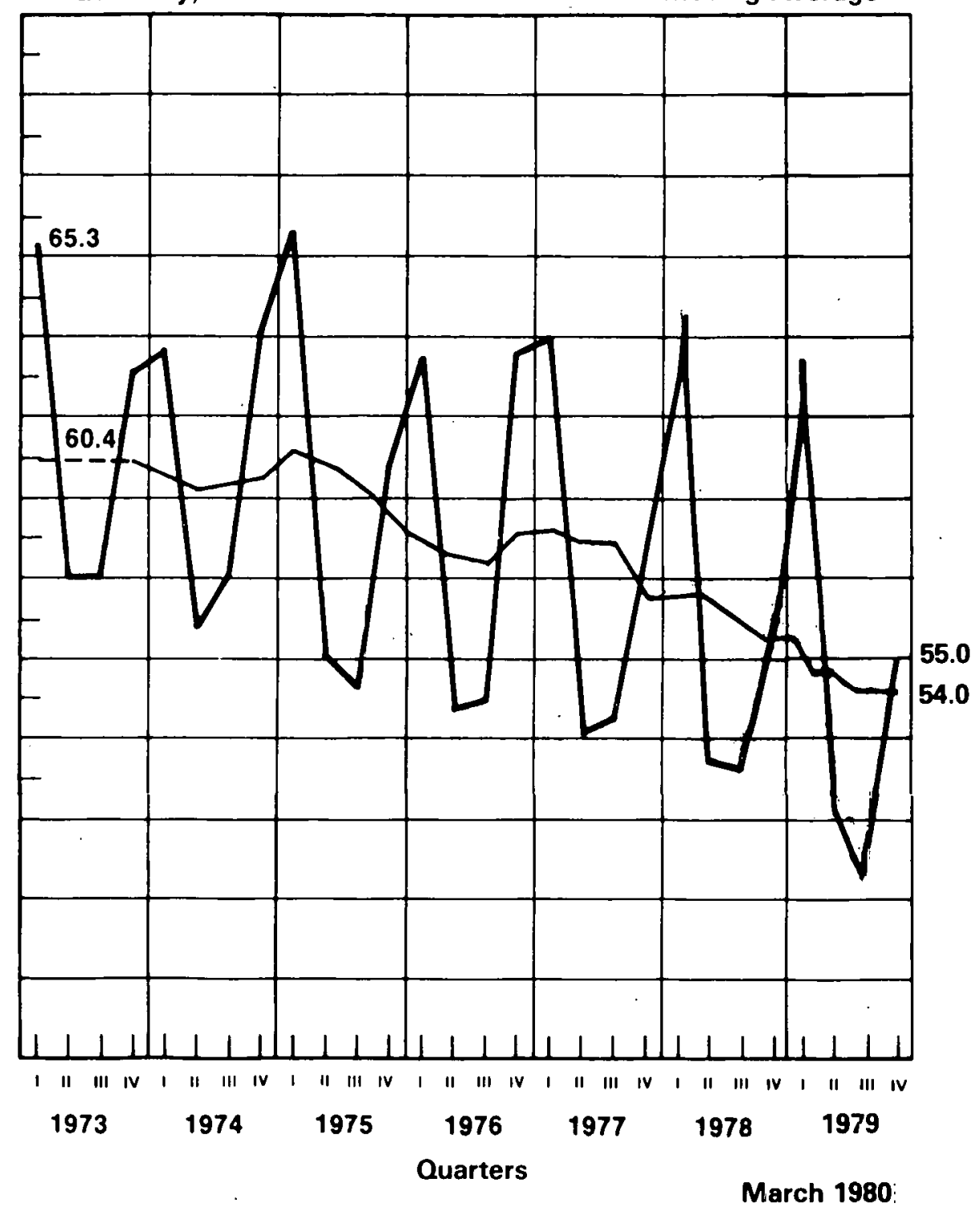




\section{United States}

Department of Energy

Washington, DC 20585

PERMIT NO. G20

FIRST CLASS MAIL

Official Business

Penalty for Private Use, $\$ 300$ 